\title{
Pinch Technique for Schwinger-Dyson Equations
}

\author{
Daniele Binosi* \\ ECT* Villa Tambosi, Strada delle Tabarelle 286, I-38050 Villazzano (Trento), Italy \\ Joannis Papavassiliou ${ }^{\dagger}$ \\ Departamento de Física Teórica and IFIC Centro Mixto, \\ Universidad de Valencia (Fundación General), \\ E-46100, Burjassot, Valencia, Spain
}

\begin{abstract}
In the context of scalar QED we derive the pinch technique self-energies and vertices directly from the Schwinger-Dyson equations. After reviewing the perturbative construction, we discuss in detail the general methodology and the basic field-theoretic ingredients necessary for the completion of this task. The construction requires the simultaneous treatment of the equations governing the scalar self-energy and the fundamental interaction vertices. The resulting non-trivial rearrangement of terms generates dynamically the Schwinger-Dyson equations for the corresponding Green's functions of the background field method. The proof relies on the extensive use of the allorder Ward-identities satisfied by the full vertices of the theory and by the one-particle-irreducible kernels appearing in the usual skeleton expansion. The Ward identities for these latter quantities are derived formally, and several subtleties related to the structure of the multiparticle kernels are addressed. The general strategy for the generalization of the method in a non-Abelian context is briefly outlined, and some of the technical difficulties are discussed.
\end{abstract}

PACS: 11.15.-q,11.15.Tk,12.38.Lg

*Electronic address: binosi@ect.it

${ }^{\dagger}$ Electronic address: joannis.papavassiliou@uv.es 


\section{INTRODUCTION}

The most widely used framework for studying in the continuum various dynamical questions that lie beyond perturbation theory are the Schwinger-Dyson equations (SDE) [1, 2]. This infinite system of coupled non-linear integral equations for all Green's functions of the theory is inherently non-perturbative, and captures the full content of the quantum equations of motion. Even though these equations are derived by an expansion about the free-field vacuum, they finally make no reference to it, or to perturbation theory, and can be used to address problems related to chiral symmetry breaking, dynamical mass generation, formation of bound states, and other non-perturbative effects [3, 4]. Since this system involves an infinite hierarchy of equations, in practice one is severely limited in their use, and the need for a self-consistent truncation scheme is evident. Devising such a scheme, however, is far from trivial, even in the case of toy models [5, 6], and becomes far more challenging when dealing with gauge theories. One of the central problems in this latter context stems from the fact that the SDEs are built out of unphysical Green's functions; thus, the extraction of reliable physical information depends crucially on delicate all-order cancellations, which may be inadvertently distorted in the process of the truncation. In QED the issues related to the truncation of the SDE are very delicate [7, 8, 9, 10, 11], but the level of complexity increases further when dealing with non-Abelian gauge theories [12], where the ghost-infested Slavnov-Taylor identities (STI) [13] make time-honored methods, such as the "gauge technique" [14], much more difficult to implement.

The truncation scheme based on the pinch technique (PT) [15] attempts to address this problem at its root, introducing a drastic modification already at the level of the building blocks of the SD series, namely the off-shell Green's functions themselves. The PT is a well-defined algorithm that exploits systematically the symmetries built into physical observables, such as $S$-matrix elements or Wilson loops, in order to construct new, effective Green's functions, endowed with very special properties, generally associated with physical observables. The basic observation, which essentially defines the PT, is that there exists a fundamental cancellation between sets of diagrams with different kinematic properties, such as self-energies, vertices, and boxes. This cancellation is driven by the underlying BRST

symmetry [16], and is triggered when a very particular subset of the longitudinal momenta circulating inside vertex and box diagrams generate out of them (by "pinching" internal 
lines) propagator-like terms. The latter are reassigned to conventional self-energy graphs, in order to give rise to the aforementioned effective Green's functions. These new Green's functions are independent of the gauge-fixing parameter [15, 17, 18, 19, 20], satisfy ghostfree, QED-like Ward identities (WI) instead of the complicated STI [17, 18], display only physical thresholds [21, 22], have correct analyticity properties [23], and are well-behaved at high energies [24]. In addition, as has been shown recently [25], the form factors of the oneloop PT three-gluon vertex [17] satisfy relations characteristic of supersymmetric scattering amplitudes. For some recent application of the PT in non-commutative theories, see [26, 27]. Returning to the SDEs, the final upshot of the PT program is to trade the conventional SD series for another, written in terms of these new Green's functions, and then truncate it, keeping only a few terms in a "dressed-loop" expansion, maintaining at the same time exact gauge-invariance [15] .

Due to various theoretical advances in recent years, the PT has been generalized to all orders in perturbation theory, both for QCD [28] and the electroweak sector of the Standard Model [29]. Of central importance in this context is the connection between the PT and the Background Field Method (BFM) [30]. The latter is a special gauge-fixing procedure that preserves the symmetry of the action under ordinary gauge transformations with respect to the background (classical) gauge field $\widehat{A}_{\mu}^{a}$, while the quantum gauge fields, $A_{\mu}^{a}$, appearing in the loops, transform homogeneously under the gauge group [31]. As a result, the background $n$-point functions satisfy QED-like all-order WIs. Note that the BFM gives rise to special Feynman rules and a characteristic ghost sector. The connection between PT and BFM [32], known to persist to all orders [28], affirms that the (gauge-independent) PT effective $n$-point functions coincide with the (gauge-dependent) BFM $n$-point functions provided that the latter are computed in the Feynman gauge.

Despite this progress, however, the truncation program outlined above is still incomplete. In fact, the direct implementation of the PT at the level of the SDE is an entirely unexplored question. Of course, PT-inspired SDEs have been treated in the literature [15, 17, 33, 34], but rather than derived they have been postulated heuristically, based on perturbative diagrammatic analysis. In the most recent work in this direction [35], the SDE for the PT gluon propagator was formulated directly in the BFM [36]; there, the connection between the PT and the BFM has been extrapolated without proof from perturbation theory to the SDE. In the present paper we take the first step toward the full implementation of the PT at 
the level of SDE. Specifically, we will carry out the PT rearrangement for the SDE of scalar QED. This Abelian model captures a plethora of the relevant conceptual issues, without the additional complications of non-Abelian theories, and serves as an excellent toy theory for gaining valuable insight on the problem.

The application of the PT in an Abelian context might seem as a trivial exercise at first, but this is certainly not the case: the self-energy of the charged scalar undergoes non-trivial pinching, displaying a great deal of the characteristics known from the nonAbelian studies. The reason for this may be traced back to a simple fact, namely the momentum dependence of the bare vertex describing the interaction between the scalars and the photon. This is exactly analogous to what happens with the three-gluon vertex, which is the central object when carrying out the PT construction in QCD. According to the standard PT methodology, from the scalar-photon vertex one isolates its "pinching" part, i.e., the combination of momenta that trigger the standard elementary WI when contracted with another such vertex. The terms generated from this WI are to be reassigned and interpreted following exactly the standard PT philosophy, arriving eventually at a new modified scalar self-energy.

The main result of this article is the following. The application of the PT at the level of the SDEs obtained in the context of the covariant gauges for the conventional Green's functions, generates dynamically the corresponding SDEs governing the BFM Green's functions. Operationally this is accomplished following the basic rules established from the perturbative analysis, sublemented by an additional crucial step. Specifically, when dealing with the SDE for the scalar self-energy, one must pinch simultaneously the SDE's governing the full vertices. It is only then that the ensuing, highly non-trivial rearrangements conspire to generate exactly the terms responsible for the conversion of the SDE for the conventional scalar self-energy into the SDE for the BFM-PT self-energy.

Instrumental for the implementation of the procedure outlined above are three ingredients: $(i)$ the all-order WI relating the divergence of the full photon-scalar vertex with the scalar self-energy; (ii) the all-order WI satisfied by the one-particle irreducible (1PI) multi-particle kernels appearing in the skeleton expansion of the SDE's for the relevant full vertices; (iii) a set of non-trivial identities [37], relating the BFM $n$-point functions to the corresponding conventional $n$-point functions in the covariant renormalizable gauges, to all orders in perturbation theory. These identities, to be referred to as Background-Quantum 
identities (BQIs), furnish a concrete field-theoretic identification of the terms that are removed during the pinching procedure from the conventional Green's functions, in order to generate their BFM counterparts [38].

The article is organized as follows. In Section $\amalg$ we review some general characteristics of scalar QED and its quantization in the conventional covariant gauges, present the all-order WI's for the two fundamental vertices, and derive formally the relevant BQIs relating the scalar self-energy and vertices in the covariant gauges with the corresponding quantities in the BFM. In section III we present a brief review of the PT applied to the case of scalar QED, and carry out explicitly the construction of the PT scalar self-energy, at one and two loops. In addition to setting up the notation and describing the general philosophy, this presentation serves as a warm-up for the generalization of the method at the level of the SDEs. Therefore, we pay particular attention to the general patterns appearing already at two loops, with special emphasis on how to reorganize various diagrams in order to identify the larger structures (Green's functions or kernels) on which the pinching momenta act. In Section IV we give a qualitative discussion of the general strategy we will follow when pinching the SDEs, comment on the technical subtleties, and determine the necessary ingredients for the implementation of this program. In Section $\nabla$ we derive in detail the all-order WI for two of the 1PI kernels appearing in the SDE's. Section VI contains the main thrust of our paper: using the machinery developed in the previous section, the PT construction is carried out explicitly for the SDE's of scalar QED. In Section VII we present our conclusions and discuss the generalization of this work to a non-Abelian context. Finally, the relevant Feynman rules are presented in an Appendix.

\section{SCALAR QED AND ITS IDENTITIES}

In this section we present the Lagrangian of scalar QED and the procedure of its gaugefixing, in the context of both conventional renormalizable gauges and BFM. We derive the all-order WI's satisfied by the fundamental vertices of the theory, and useful identities (BQI)

relating the Green's functions of the theory in the two aforementioned gauge-fixing schemes. 


\section{A. Lagrangian and gauge fixing}

We will concentrate on scalar QED, which describes a complex scalar field $\phi$ interacting with the electromagnetic field $A_{\mu}$. The Lagrangian density is

$$
\mathcal{L}=\mathcal{L}_{\mathrm{I}}+\mathcal{L}_{\mathrm{GF}}+\mathcal{L}_{\mathrm{FPG}},
$$

with $\mathcal{L}_{\mathrm{I}}$ the gauge invariant $U(1)$ Lagrangian,

$$
\mathcal{L}_{\mathrm{I}}=-\frac{1}{4} F^{\mu \nu} F_{\mu \nu}+\left(\mathcal{D}^{\mu} \phi\right)^{\dagger}\left(\mathcal{D}_{\mu} \phi\right)-m^{2} \phi^{\dagger} \phi+\frac{\lambda}{4}\left(\phi^{\dagger} \phi\right)^{2},
$$

where the field strength is

$$
F_{\mu \nu}=\partial_{\mu} A_{\nu}-\partial_{\nu} A_{\mu}
$$

and the covariant derivative is defined as

$$
\mathcal{D}_{\mu}=\partial_{\mu}-i g Y_{\phi} A_{\mu}
$$

with $g$ the coupling constant and $Y_{\phi}$ the scalar field hypercharge $\left(Y_{\phi}=1\right)$. In general, the (covariant) gauge fixing term and the Faddeev-Popov ghost can be written as

$$
\begin{aligned}
\mathcal{L}_{\mathrm{GF}} & =\frac{\xi}{2} B^{2}+B \mathcal{F}, \\
\mathcal{L}_{\mathrm{FPG}} & =-\bar{c} s \mathcal{F} .
\end{aligned}
$$

In the formulas above, $\mathcal{F}$ is the gauge fixing function, $B$ is an auxiliary, non-dynamical field that can be eliminated through its (trivial) equation of motion, $c(\bar{c})$ is the ghost (anti-ghost) field, while $s$ is the BRST operator, with

$$
\begin{array}{rl}
s A_{\mu}=\partial_{\mu} c & s \phi=i g c \phi, \\
s \phi^{\dagger}=-i g c \phi^{\dagger} & s c=0, \\
s \bar{c}=B & s B=0 .
\end{array}
$$

In view of the equivalence between the PT Green's functions and the BFM ones at $\xi_{\mathrm{Q}}=1$ we will consider the following two gauge fixing procedures (the corresponding Feynman rules relevant for our calculation are given in the Appendix).

1. In the usual $R_{\xi}$ gauges, one chooses $\mathcal{F}=\partial^{\mu} A_{\mu}$, to get

$$
\begin{aligned}
\mathcal{L}_{\mathrm{GF}} & =-\frac{1}{2 \xi}\left(\partial^{\mu} A_{\mu}\right)^{2}, \\
\mathcal{L}_{\mathrm{FPG}} & =-\bar{c} \partial^{2} c .
\end{aligned}
$$

In this gauge the ghosts are, of course, decoupled and play no dynamical role. 
2. In the case of the BFM, one splits the scalar field into a background part, $\widehat{\phi}$, and its quantum part, $\phi$. Notice that the BRST variation of the background field will be zero, but the latter will enter in the variation of the quantum one, i.e.,

$$
\begin{array}{rl}
s \phi=i g c(\widehat{\phi}+\phi) & s \phi^{\dagger}=-i g c\left(\widehat{\phi}^{\dagger}+\phi^{\dagger}\right) \\
s \widehat{\phi}=0 & s \widehat{\phi}^{\dagger}=0 .
\end{array}
$$

In this case the gauge fixing function is

$$
\mathcal{F}=\partial^{\mu} A_{\mu}-i g \xi\left(\widehat{\phi}^{\dagger} \phi-\phi^{\dagger} \widehat{\phi}\right)
$$

which gives in turn

$$
\begin{aligned}
\mathcal{L}_{\mathrm{GF}} & =-\frac{1}{2 \xi}\left(\partial^{\mu} A_{\mu}\right)^{2}+i g \partial^{\mu} A_{\mu}\left(\widehat{\phi}^{\dagger} \phi-\phi^{\dagger} \widehat{\phi}\right) \\
& +\frac{g^{2}}{2} \xi\left[\left(\widehat{\phi}^{\dagger} \phi\right)^{2}+\left(\phi^{\dagger} \widehat{\phi}\right)^{2}-2 \widehat{\phi}^{\dagger} \phi \phi^{\dagger} \widehat{\phi}\right], \\
\mathcal{L}_{\mathrm{FPG}} & =-\bar{c} \partial^{2} c-g^{2} \xi \bar{c}\left(\widehat{\phi}^{\dagger} c \phi+2 \widehat{\phi}^{\dagger} c \widehat{\phi}+\phi^{\dagger} c \widehat{\phi}\right) .
\end{aligned}
$$

Therefore, in the BFM ghosts are not decoupled. In order to obtain the full set of Feynman rules in this gauge, one needs also to consider the extra terms coming from the background-quantum splitting carried out inside the gauge invariant Lagrangian, i.e., $\mathcal{L}_{\mathrm{I}}\left(\phi, \phi^{\dagger}\right) \rightarrow \mathcal{L}_{\mathrm{I}}\left(\widehat{\phi}+\phi, \widehat{\phi}^{\dagger}+\phi^{\dagger}\right)$, see again the Appendix.

\section{B. Some fundamental identities}

In this subsection we review briefly the Batalin-Vilkovisky formalism [39], which allows one to get simultaneously both the WIs as well as the BQIs of our theory.

Let us then start by introducing for each field $\Phi$ appearing in the theory the corresponding anti-field, to be denoted by $\Phi^{*}$. The anti-field $\Phi^{*}$ has opposite statistics with respect to $\Phi$; its ghost number, $\operatorname{gh}\left(\Phi^{*}\right)$, is related to the ghost number $\operatorname{gh}(\Phi)$ of the field $\Phi$ by $\operatorname{gh}\left(\Phi^{*}\right)=$ $-1-\operatorname{gh}(\Phi)$. The ghost numbers of the various fields and anti-fields are summarized in table I. Next, we add to the original gauge invariant Lagrangian a term coupling the anti-fields with the BRST variation of the corresponding fields, to get

$$
\begin{aligned}
\mathcal{L}_{\mathrm{BV}} & =\mathcal{L}_{\mathrm{I}}+\mathcal{L}_{\mathrm{BRST}} \\
& =\mathcal{L}_{\mathrm{I}}+\sum_{\Phi} \Phi^{*} s \Phi
\end{aligned}
$$




\begin{tabular}{|c|c|c|c|c||c|c|c|c|}
\hline$\Phi$ & $A_{\mu}$ & $\phi$ & $c$ & $\bar{c}$ & $A_{\mu}^{*}$ & $\phi^{*}$ & $c^{*}$ & $\bar{c}^{*}$ \\
\hline $\operatorname{gh}(\Phi)$ & 0 & 0 & 1 & -1 & -1 & -1 & 0 & -2 \\
\hline
\end{tabular}

TABLE I: The ghost numbers of the fields and antifields in scalar QED.

As a consequence of the BRST invariance of the action and the nilpotency of the BRST operator, the action $\Gamma^{(0)}\left[\Phi, \Phi^{*}\right]$ constructed from $\mathcal{L}_{\mathrm{BV}}$, will satisfy the master equation

$$
\int d^{4} x \sum_{\Phi} \frac{\delta \Gamma^{(0)}}{\delta \Phi^{*}} \frac{\delta \Gamma^{(0)}}{\delta \Phi}=0 .
$$

Since the anti-fields are external sources, we must constrain them to suitable values before we use the action $\Gamma^{(0)}$ in $S$-matrix elements calculations [31]. To that end, we introduce an arbitrary fermionic functional $\Psi[\Phi]$ (also referred to as "gauge fixing fermion", for reasons that will become clear shortly), such that

$$
\Phi^{*}=\frac{\delta \Psi[\Phi]}{\delta \Phi}
$$

Then the action becomes

$$
\begin{aligned}
\Gamma^{(0)}[\phi, \delta \Psi / \delta \Phi] & =\Gamma^{(0)}[\Phi]+(s \Phi) \frac{\delta \Psi[\Phi]}{\delta \Phi} \\
& =\Gamma^{(0)}[\Phi]+s \Psi[\Phi]
\end{aligned}
$$

and choosing the functional $\Psi$ to satisfy the relation

$$
s \Psi=\int d^{4} x\left(\mathcal{L}_{\mathrm{GF}}+\mathcal{L}_{\mathrm{FPG}}\right),
$$

we see that the action $\Gamma^{(0)}$ (obtained from $\mathcal{L}_{\mathrm{BV}}$ ) is equivalent to the gauge fixed action obtained from the original Lagrangian $\mathcal{L}$ of Eq.(2.1). Furthermore, the antighost anti-field $\bar{c}^{*}$ and the multiplier $B$ enter bi-linearly in the action, and one can write

$$
\Gamma^{(0)}\left[\Phi, \Phi^{*}\right]=\Gamma_{\min }^{(0)}\left[A_{\mu}, A_{\mu}^{*}, \phi, \phi^{*}, \phi^{\dagger}, \phi^{* \dagger}, c, c^{*}\right]-B \bar{c}^{*}
$$

with $\Gamma_{\min }^{(0)}$ satisfying the master equation (2.16) by itself. In what follows we will restrict our considerations to the minimal action, dropping the corresponding subscript.

The quantum corrected version of the master equation (2.16) is established in the form of the WI functional

$$
\mathcal{S}(\Gamma)\left[\Phi, \Phi^{*}\right]=\int d^{4} x \sum_{\Phi} \frac{\delta \Gamma}{\delta \Phi^{*}} \frac{\delta \Gamma}{\delta \Phi}=0
$$


where $\Gamma\left[\Phi, \Phi^{*}\right]$ is now the effective action. The equation above must hold in any theory with a unitary $S$-matrix and gauge-independent physical observables, and gives rise to the complete set of the all-order WIs, via the repeated application of functional differentiations, keeping in mind that: $(i) \mathcal{S}(\Gamma)$ has ghost charge 1; (ii) functions with non-zero ghost charge vanish (since the ghost charge is a conserved quantity); (iii) the BRST transformation of the gauge field is proportional to the ghost $s A_{\mu}=\partial_{\mu} c$. Overall, these latter observations imply that in order to extract non-zero identities from Eq.(2.21) one needs to differentiate the latter with respect to one ghost filed, or two ghost fields and one anti-field (the only exception to this rule is when differentiating with respect to a ghost anti-field.) In particular, identities involving one or more gauge fields are obtained differentiating Eq.(2.21) with respect to the set of fields in which one gauge boson has been replaced by the corresponding ghost field.

In the remainder of this section we will adopt for the $n$-point Green's function the notation

$$
\Gamma_{\Phi_{1} \Phi_{2} \ldots \Phi_{n-1} \Phi_{n}}\left(p_{2}, \ldots, p_{n-1}, p_{n}\right)=\left.i^{n} \frac{\delta^{n} \Gamma}{\delta \Phi_{1}\left(p_{1}\right) \delta \Phi_{2}\left(p_{2}\right) \cdots \delta \Phi_{n}\left(p_{n}\right)}\right|_{\Phi_{i}=0},
$$

with $p_{i}$ the in-going momentum of the $\Phi_{i}$ field. The momentum for the field $\Phi_{1}$ follows from momentum conservation, and we will not write it explicitly.

\section{Ward Identities}

We begin with the STI for the photon-scalar-scalar vertex. From our previous discussion follows that, for obtaining such an identity, one needs to consider the functional differentiation

$$
\left.\frac{\delta^{3} \mathcal{S}(\Gamma)}{\delta \phi^{\dagger}\left(p_{1}\right) \delta \phi\left(p_{2}\right) \delta c(q)}\right|_{\Phi=0}=0 \quad p_{1}+p_{2}+q=0 .
$$

Carrying out the functional differentiation we obtain the equation

$$
\Gamma_{c A_{\mu}^{*}}(q) \Gamma_{A_{\mu} \phi^{\dagger} \phi}\left(p_{1}, p_{2}\right)+\Gamma_{\phi^{\dagger} c \phi^{*}}\left(q, p_{2}\right) \Gamma_{\phi^{\dagger} \phi}\left(p_{2}\right)+\Gamma_{\phi c \phi^{* \dagger}}\left(q, p_{1}\right) \Gamma_{\phi \phi^{\dagger}}\left(p_{1}\right)=0
$$

On the other hand, the Abelian nature of the theory, together with the decoupling of the ghosts in the $R_{\xi}$ gauges, enforce the validity of the following (all order) equations (see also the Appendix)

$$
\begin{aligned}
& \Gamma_{c A_{\mu}^{*}}(q)=\Gamma_{c A_{\mu}^{*}}^{(0)}(q)=-q^{\mu}, \\
& \Gamma_{\phi^{\dagger} c \phi^{*}}\left(q, p_{2}\right)=\Gamma_{\phi^{\dagger} c \phi^{*}}^{(0)}\left(q, p_{2}\right)=g, \\
& \Gamma_{\phi c \phi^{* \dagger}}\left(q, p_{1}\right)=\Gamma_{\phi c \phi^{* \dagger}}^{(0)}\left(q, p_{1}\right)=-g,
\end{aligned}
$$


which furnish the fundamental WI

$$
q^{\mu} \Gamma_{A_{\mu} \phi^{\dagger} \phi}\left(p_{1}, p_{2}\right)=g\left[\Gamma_{\phi \phi^{\dagger}}\left(p_{2}\right)-\Gamma_{\phi \phi^{\dagger}}\left(p_{1}\right)\right] .
$$

Introducing the short-hand notation $\Gamma_{A_{\mu} \phi^{\dagger} \phi} \equiv \Gamma_{\mu}$ and $\Gamma_{\phi \phi^{\dagger}} \equiv S^{-1}$, we finally get

$$
q^{\mu} \Gamma_{\mu}\left(p_{1}, p_{2}\right)=g\left[S^{-1}\left(p_{2}\right)-S^{-1}\left(p_{1}\right)\right]
$$

Let us derive next the WI satisfied by the 4-point function $\Gamma_{A_{\mu} A_{\nu} \phi^{\dagger} \phi}$. In this case we need to consider the functional differentiation

$$
\left.\frac{\delta^{4} \mathcal{S}(\Gamma)}{\delta \phi^{\dagger}\left(p_{1}\right) \delta \phi\left(p_{2}\right) \delta A_{\nu}(k) \delta c(q)}\right|_{\Phi=0}=0 \quad p_{1}+p_{2}+k+q=0 .
$$

Carrying out the functional differentiation, and considering that, due to the decoupling of the ghost fields, one has to all orders

$$
\begin{array}{cl}
\Gamma_{A_{\nu} c A_{\mu}^{*}}=0 & \Gamma_{\phi^{\dagger} \phi c A_{\mu}^{*}}=0, \\
\Gamma_{\phi^{\dagger} A_{\nu} c \phi^{*}}=0 & \Gamma_{\phi A_{\nu} c \phi^{* \dagger}}=0,
\end{array}
$$

we obtain

$$
\begin{aligned}
\Gamma_{c A_{\mu}^{*}}(q) \Gamma_{A_{\mu} A_{\nu} \phi^{\dagger} \phi}\left(k, p_{1}, p_{2}\right) & +\Gamma_{\phi^{\dagger} c \phi^{*}}\left(q, k+p_{2}\right) \Gamma_{\phi^{\dagger} A_{\nu} \phi}\left(k, p_{2}\right) \\
& +\Gamma_{\phi c \phi^{* \dagger}}\left(q, k+p_{1}\right) \Gamma_{\phi A_{\nu} \phi^{\dagger}}\left(k, p_{1}\right)=0 .
\end{aligned}
$$

Then, using once again the results of Eq.(2.25), one gets the final identity

$$
\begin{aligned}
q^{\mu} \Gamma_{A_{\mu} A_{\nu} \phi^{\dagger} \phi}\left(k, p_{1}, p_{2}\right) & =g\left[\Gamma_{\phi^{\dagger} A_{\nu} \phi}\left(k, p_{2}\right)-\Gamma_{\phi A_{\nu} \phi^{\dagger}}\left(k, p_{1}\right)\right] \\
& =g\left[\Gamma_{A_{\nu} \phi^{\dagger} \phi}\left(-k-p_{2}, p_{2}\right)-\Gamma_{A_{\nu} \phi^{\dagger} \phi}\left(p_{1},-k-p_{1}\right)\right],
\end{aligned}
$$

which can be rewritten as

$$
q^{\mu} \Gamma_{\mu \nu}\left(k, p_{1}, p_{2}\right)=g\left[\Gamma_{\nu}\left(-k-p_{2}, p_{2}\right)-\Gamma_{\nu}\left(p_{1},-k-p_{1}\right)\right]
$$

where we have set $\Gamma_{A_{\mu} A_{\nu} \phi^{\dagger} \phi} \equiv \Gamma_{\mu \nu}$.

\section{Background quantum identities}

Background quantum identities are identities that relate Green's functions involving background fields to Green's functions involving only quantum ones. Therefore, they are particularly useful in the PT context, since they allow for a direct comparison between PT and BFM Green's functions. 
To obtain such identities for scalar QED, we introduce a classical scalar field $\Omega^{\phi}$ and its complex conjugate $\Omega^{\phi^{\dagger}}$, carrying the same quantum numbers as the scalar, but with ghost charge +1 . We then implement the equation of motion of the background fields at the quantum level, by extending the BRST symmetry through the equations

$$
\begin{aligned}
s \widehat{\phi}=\Omega^{\phi} & s \Omega^{\phi}=0, \\
s \widehat{\phi^{\dagger}}=\Omega^{\phi^{\dagger}} & s \Omega^{\phi^{\dagger}}=0 .
\end{aligned}
$$

The dependence of the Green's function on the background field is then controlled by the modified STI functional

$$
\begin{aligned}
\mathcal{S}^{\prime}\left(\Gamma^{\prime}\right)\left[\Phi, \Phi^{*}\right] & =\mathcal{S}\left(\Gamma^{\prime}\right)\left[\Phi, \Phi^{*}\right]+\int d^{4} x\left[\Omega^{\phi}\left(\frac{\delta \Gamma^{\prime}}{\delta \widehat{\phi}}-\frac{\delta \Gamma^{\prime}}{\delta \phi}\right)+\Omega^{\phi^{\dagger}}\left(\frac{\delta \Gamma^{\prime}}{\delta \widehat{\phi}^{\dagger}}-\frac{\delta \Gamma^{\prime}}{\delta \phi^{\dagger}}\right)\right] \\
& =\int d^{4} x\left\{\frac{\delta \Gamma^{\prime}}{\delta A_{\mu}^{*}} \frac{\delta \Gamma^{\prime}}{\delta A_{\mu}}+\frac{\delta \Gamma^{\prime}}{\delta c^{*}} \frac{\delta \Gamma^{\prime}}{\delta c}+\frac{\delta \Gamma^{\prime}}{\delta \phi^{*}} \frac{\delta \Gamma^{\prime}}{\delta \phi^{\dagger}}+\frac{\delta \Gamma^{\prime}}{\delta \phi^{\dagger *}} \frac{\delta \Gamma^{\prime}}{\delta \phi}\right. \\
& \left.+\left[\Omega^{\phi}\left(\frac{\delta \Gamma^{\prime}}{\delta \widehat{\phi}}-\frac{\delta \Gamma^{\prime}}{\delta \phi}\right)+\Omega^{\phi^{\dagger}}\left(\frac{\delta \Gamma^{\prime}}{\delta \widehat{\phi}^{\dagger}}-\frac{\delta \Gamma^{\prime}}{\delta \phi^{\dagger}}\right)\right]\right\},
\end{aligned}
$$

where $\Gamma^{\prime}$ denotes the effective action that depends on the background sources $\left(\left.\Gamma \equiv \Gamma^{\prime}\right|_{\Omega=0}\right)$. Differentiation of the above functional with respect to background sources and background and/or quantum fields will then relate 1PI functions involving different background/quantum field content.

The first BQI we derive involves two background scalar fields. One begins by considering the functional differentiations

$$
\begin{array}{ll}
\left.\frac{\delta^{2} \mathcal{S}^{\prime}\left(\Gamma^{\prime}\right)}{\delta \Omega^{\phi^{\dagger}}\left(p_{1}\right) \delta \widehat{\phi}(q)}\right|_{\Phi=0}=0 & q+p_{1}=0, \\
\left.\frac{\delta^{2} \mathcal{S}^{\prime}\left(\Gamma^{\prime}\right)}{\delta \Omega^{\phi^{\dagger}}\left(p_{1}\right) \delta \phi(q)}\right|_{\Phi=0}=0 & q+p_{1}=0
\end{array}
$$

which furnish the intermediate BQIs

$$
\begin{aligned}
& \Gamma_{\widehat{\phi} \bar{\phi}^{\dagger}}(q)=\Gamma_{\widehat{\phi} \phi^{\dagger}}(q)+\Gamma_{\Omega^{\phi^{\dagger} \phi^{*}}}(q) \Gamma_{\widehat{\phi} \phi^{\dagger}}(q)+\Gamma_{\Omega^{\phi^{\dagger}} A_{\mu}^{*}}(q) \Gamma_{\widehat{\phi} A_{\mu}}(q), \\
& \Gamma_{\widehat{\phi} \phi^{\dagger}}(q)=\Gamma_{\phi \phi^{\dagger}}(q)+\Gamma_{\Omega^{\phi} \phi^{* \dagger}}(q) \Gamma_{\phi \phi^{\dagger}}(q)+\Gamma_{\Omega^{\phi} A_{\mu}^{*}}(q) \Gamma_{\phi^{\dagger} A_{\mu}}(q) .
\end{aligned}
$$

According to our previous discussion, in the above equation all Green's function involving ghost legs have dropped out (having ghost charge different from zero). The Abelian nature of the theory enforces to all orders the identity

$$
\Gamma_{\Omega^{\phi^{\dagger} A_{\mu}^{*}}}(q)=\Gamma_{\Omega^{\phi} A_{\mu}^{*}}(q)=0
$$


so that the BQI relating the background 1PI two-point function to the quantum one reads

$$
\Gamma_{\widehat{\phi} \bar{\phi}^{\dagger}}(q)=\left[1+\Gamma_{\Omega^{\phi^{\dagger} \phi^{*}}}(q)\right]^{2} \Gamma_{\phi \phi^{\dagger}}(q) .
$$

Introducing the auxiliary function $G \equiv \Gamma_{\Omega^{\phi^{\dagger}} \phi^{*}}$, and denoting $\Gamma_{\widehat{\phi} \widehat{\phi}^{\dagger}} \equiv \widehat{S}^{-1}$, we obtain the BQI in its final form,

$$
\widehat{S}^{-1}(q)=[1+G(q)]^{2} S^{-1}(q)
$$

Notice that, in the Abelian case, the 1PI Green's function $G$ has a particularly simple expression, namely

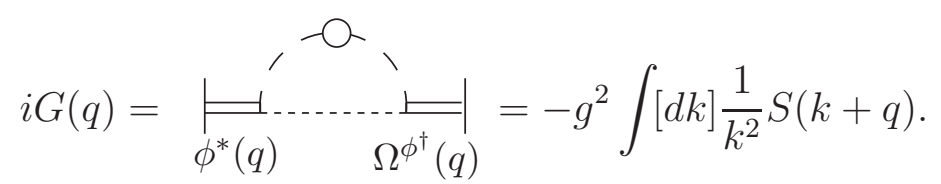

In the diagram above (and all those that follow) we use the graphic notation and the Feynman rules described in the Appendix, and denote with white (respectively black) blobs connected (respectively one particle irreducible) Green's functions.

Next, we need the BQI relating the trilinear vertex with all quantum fields to the vertex where a (quantum) scalar field has been replaced by a background one. To this end, we consider the functional differentiation

$$
\left.\frac{\delta^{3} \mathcal{S}^{\prime}\left(\Gamma^{\prime}\right)}{\delta A_{\mu}\left(k_{1}\right) \delta \Omega^{\phi}(q) \delta \phi^{\dagger}\left(p_{1}\right)}\right|_{\Phi=0}=0 \quad q+p_{1}+k_{1}=0 .
$$

Then, taking into account that, for the model at hand, all functions involving the combination $\Omega^{\phi} A_{\mu}^{*}$ are a fortiori $1 \mathrm{PR}$, and therefore drop out from the identity, we find the identity

$$
\Gamma_{A_{\mu} \phi^{\dagger} \widehat{\phi}}\left(p_{1}, q\right)=\left[1+\Gamma_{\Omega^{\phi} \phi^{* \dagger}}(q)\right] \Gamma_{A_{\mu} \phi^{\dagger} \phi}\left(p_{1}, q\right)+\Gamma_{A_{\mu} \Omega^{\phi} \phi^{* \dagger}}\left(p_{1}, q\right) \Gamma_{\phi \phi^{\dagger}}\left(p_{1}\right) .
$$

Introducing the notation $\widehat{\Gamma}_{\mu} \equiv \Gamma_{A_{\mu} \phi^{\dagger} \widehat{\phi}}$ and $G_{\mu} \equiv \Gamma_{A_{\mu} \Omega^{\phi} \phi^{* \dagger}}$, we can cast the above BQI in a short-hand form

$$
\widehat{\Gamma}_{\mu}\left(p_{1}, q\right)=[1+G(q)] \Gamma_{\mu}\left(p_{1}, q\right)+G_{\mu}\left(p_{1}, q\right) S^{-1}\left(p_{1}\right)
$$

Notice that the function $G_{\mu}\left(q, p_{1}\right)$ has the simple expression

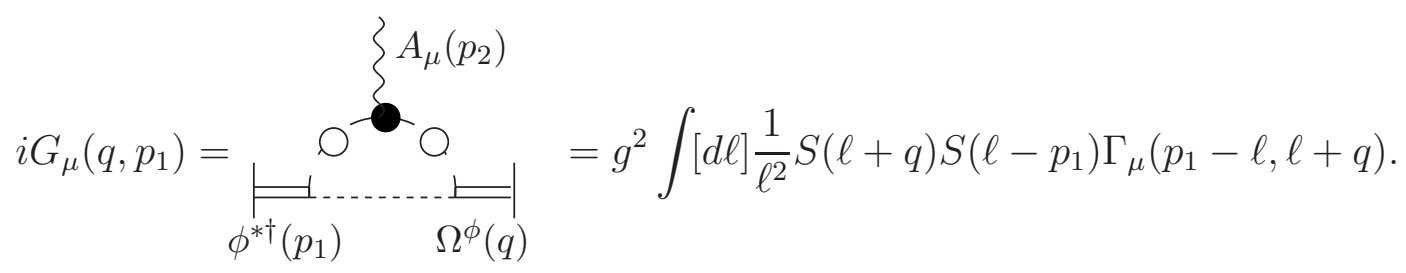


In order to obtain the BQI for the quadrilinear vertex one needs to consider the functional differentiation

$$
\left.\frac{\delta^{4} \mathcal{S}^{\prime}\left(\Gamma^{\prime}\right)}{\delta A_{\mu}\left(k_{1}\right) \delta A_{\nu}\left(k_{2}\right) \delta \Omega^{\phi}(q) \delta \phi^{\dagger}\left(p_{1}\right)}\right|_{\Phi=0}=0 \quad q+p_{1}+k_{1}+k_{2}=0,
$$

which provides the BQI

$$
\begin{aligned}
\Gamma_{A_{\mu} A_{\nu} \phi^{\dagger} \widehat{\phi}}\left(k_{2}, p_{1}, q\right) & =\left[1+\Gamma_{\Omega^{\phi} \phi^{* \dagger}}(q)\right] \Gamma_{A_{\mu} A_{\nu} \phi^{\dagger} \phi}\left(k_{2}, p_{1}, q\right)+\Gamma_{A_{\mu} A_{\nu} \Omega^{\phi} \phi^{* \dagger}}\left(k_{2}, q, p_{1}\right) \Gamma_{\phi^{\dagger} \phi}\left(p_{1}\right) \\
& +\Gamma_{A_{\mu} \Omega^{\phi} \phi^{* \dagger}}\left(q, k_{2}+p_{1}\right) \Gamma_{A_{\nu} \phi^{\dagger} \phi}\left(p_{1},-p_{1}-k_{2}\right) \\
& +\Gamma_{A_{\nu} \Omega^{\phi} \phi^{* \dagger}}\left(q, k_{1}+p_{1}\right) \Gamma_{A_{\mu} \phi^{\dagger} \phi}\left(p_{1},-p_{1}-k_{1}\right)
\end{aligned}
$$

Introducing the notation $\widehat{\Gamma}_{\mu \nu} \equiv \Gamma_{A_{\mu} A_{\nu} \phi^{\dagger} \widehat{\phi}}$ and $G_{\mu \nu} \equiv \Gamma_{A_{\mu} A_{\nu} \Omega^{\phi} \phi^{* \dagger}}$ we can rewrite the above BQI in its final form, namely

$$
\begin{aligned}
\widehat{\Gamma}_{\mu \nu}\left(k_{2}, p_{1}, q\right) & =[1+G(q)] \Gamma_{\mu \nu}\left(k_{2}, p_{1}, q\right)+G_{\mu \nu}\left(k_{2}, q, p_{1}\right) S^{-1}\left(p_{1}\right) \\
& +G_{\mu}\left(q, k_{2}+p_{1}\right) \Gamma_{\nu}\left(p_{1},-p_{1}-k_{2}\right)+G_{\nu}\left(q, k_{1}+p_{1}\right) \Gamma_{\mu}\left(p_{1},-p_{1}-k_{1}\right) .
\end{aligned}
$$

The equation for the auxiliary function $G_{\mu \nu}$ is given by

$$
i G_{\mu \nu}\left(k_{2}, q, p_{1}\right)=\underset{\phi^{* \dagger}\left(p_{1}\right)}{\left.A_{\mu}\left(k_{1}\right)\right\}} \int_{\Omega^{\phi}(q)} \int_{A_{\nu}\left(k_{2}\right)}=g^{2} \int[d \ell] \frac{1}{\ell^{2}} S(\ell+q) S\left(\ell-p_{1}\right) \mathcal{C}_{\mu \nu}\left(k_{2}, p_{1}-\ell, \ell+q\right),
$$

where $\mathcal{C}_{\mu \nu}$ is the four-particle connected Green's function with two photons and two scalar entering, whose properties and WI will be discussed in detail later.

Finally, we report for completeness the WI satisfied by the auxiliary functions $G_{\mu}$ and $G_{\mu \nu}$. Contracting directly their defining equations, Eq. (2.45) and Eq. (2.48), using Eq. (2.27), and the WI for the kernel $\mathcal{C}_{\mu \nu}$ derived in Section V, Eq. (5.12), we obtain

$$
\begin{aligned}
p_{2}^{\mu} G_{\mu}\left(q, p_{1}\right) & =g\left[G(q)-G\left(p_{1}\right)\right], \\
k_{1}^{\mu} G_{\mu \nu}\left(k_{2}, q, p_{1}\right) & =g\left[G_{\nu}\left(q+k_{1}, p_{1}\right)-G_{\nu}\left(q, p_{1}+k_{1}\right)\right] .
\end{aligned}
$$

\section{PT IN SCALAR QED: GENERAL CONSIDERATIONS}

In this section we present the general methodology for constructing PT self-energies in the case of scalar QED. The object of interest will be the scalar self-energy: due to the 
momenta appearing in the elementary vertices, the PT algorithm allows the conversion of the standard scalar self-energy into the BFM scalar self-energy, e.g., with a background scalar entering and exiting. Note that the photon self-energy remains intact, because no WI can be triggered within the corresponding graphs defining it. After outlining the general philosophy and setting up some useful notation, we will proceed to review the construction of PT scalar self-energy at one and two loops.

The general idea of the PT is to identify, following a very strict procedure, propagator-like contributions contained in vertex- and box-diagrams, and reassign them to the conventional self-energy graphs [15], thus generating new, effective Green's functions, with special properties. This construction is carried out inside an $S$-matrix element, or some other gaugeinvariant observable; the underlying symmetries, most notably the BRST symmetry, enforce crucial cancellations, making the aforementioned construction possible. The "S-matrix" PT described above has an equivalent version, known as "intrinsic" PT [17]. According to it, one identifies the parts of the self-energy that will cancel against the pinching terms coming from vertices and boxes, and discards them directly from the self-energy: what remains is the answer. The intrinsic PT is operationally more economical, and minimizes the need of embedding the procedure into a physical observable; in what follows we will adopt this latter approach.

The rearangments of graphs mentioned above are realized when judiciously selected longitudinal momenta, circulating inside the Feynman graphs, trigger elementary WI's. These momenta stem either from the longitudinal (gauge-dependent) parts of the propagators, or from parts of the momenta carried by the "external" elementary vertices [40] i.e., vertices where the physical momentum enters or leaves the corresponding diagram. The construction is simplified enormously in the context of the renormalizable gauges, if one chooses directly the Feynman gauge [17]. This choice eliminates all pinching momenta, other than those stemming from the external vertices. Such a choice constitutes no loss of generality, as has been explained in [29], by establishing a close correspondence between the PT and the powerful Nielsen identities [41] that control the gauge-dependence of the conventional Green's functions.

Let us now turn to the case of scalar QED, and outline the construction of the PT scalar 


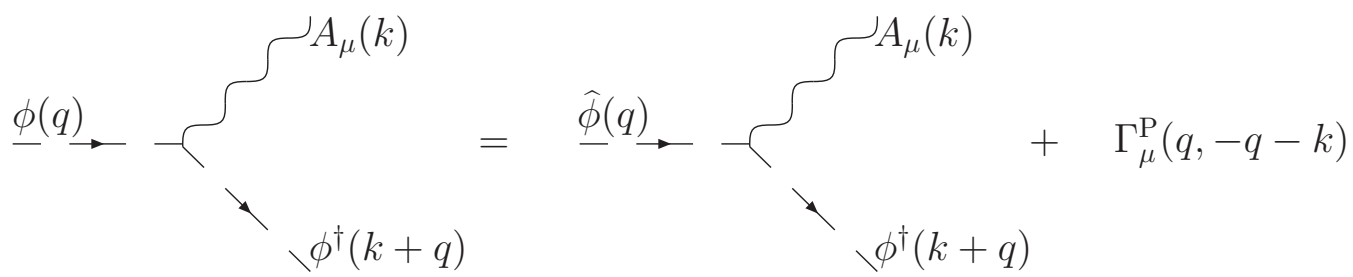

FIG. 1: The PT decomposition of the elementary vertex

self-energy. The bare photon propagator, $\Delta_{\mu \nu}^{(0)}(k)$, assumes the form

$$
\Delta_{\mu \nu}^{(0)}(k)=-\frac{i}{k^{2}}\left[g_{\mu \nu}-(1-\xi) \frac{k_{\mu} k_{\nu}}{k^{2}}\right],
$$

and, following the previous discussion, we will choose directly the Feynman gauge, $\xi=1$. Therefore, the only pinching momenta will originate from the bare (tree-level) scalar-scalarphoton vertex $\Gamma_{\mu}^{(0)}$. According to the PT, this latter vertex is to be split into two parts: $(i)$ a part, to be denoted by $\Gamma^{\mathrm{P}}$, which contains longitudinal momenta, i.e., momenta that can be contracted with the vertex on the other side of the diagram, thus triggering an elementary WI, and $(i i)$ the remainder, to be denoted by $\Gamma^{\mathrm{F}}$, which coincides with the corresponding tree-level vertex in the BFM; in particular, the background field is to be identified with the field carrying the external momentum. In the case of the scalar vertex $\Gamma_{\mu}^{(0)}(q,-q-k)$, the only longitudinal momentum is $k_{\mu}$, irrigating the photon line; therefore, the PT decomposition of the vertex described above amounts to (see also Fig 1)

$$
\Gamma_{\mu}^{(0)}(q,-q-k)=\Gamma_{\mu}^{\mathrm{F}}(q,-q-k)+\Gamma_{\mu}^{\mathrm{P}}(q,-q-k),
$$

with

$$
\begin{aligned}
i \Gamma_{\mu}^{(0)}(q,-q-k) & =-i g(2 q+k)_{\mu}, \\
i \Gamma_{\mu}^{\mathrm{F}}(q,-q-k) & =-2 i g q_{\mu}, \\
i \Gamma_{\mu}^{\mathrm{P}}(q,-q-k) & =-i g k_{\mu} .
\end{aligned}
$$

For the case of the scalar self-energy, the above splitting is to be carried out to the two external vertices, where the physical momentum $q$ is entering and exiting: Specifically, we write

$$
\Gamma_{\mu}^{(0)}[\ldots] \Gamma_{\nu}^{(0)}=\Gamma_{\mu}^{\mathrm{F}}[\ldots] \Gamma_{\nu}^{\mathrm{F}}+\Gamma_{\mu}^{\mathrm{P}}[\ldots] \Gamma_{\nu}^{(0)}+\Gamma_{\mu}^{(0)}[\ldots] \Gamma_{\nu}^{\mathrm{P}}-\Gamma_{\mu}^{\mathrm{P}}[\ldots] \Gamma_{\nu}^{\mathrm{P}} .
$$

where $[\ldots]$ denotes the rest of the diagram appearing between the two vertices. In what follows we will use the short-hand notation $[d k]=\mu^{\epsilon} d^{d} k /(2 \pi)^{d}$, with $d=4-\epsilon$, the dimension 


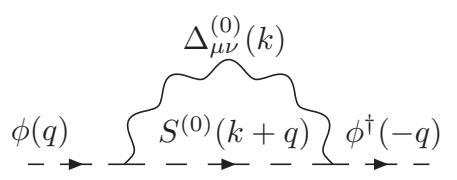

(a)

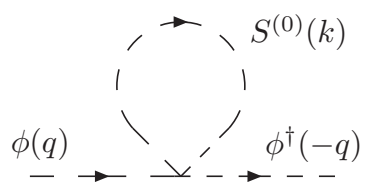

(b)

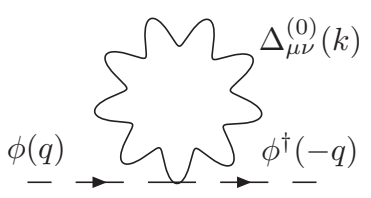

(c)

FIG. 2: The diagrams which, when evaluated using the corresponding Feynman rules (see Appendix, Fig 10), contribute at one loop to the conventional and BFM scalar self-energies, $\Sigma^{(1)}(q)$ and $\widehat{\Sigma}^{(1)}(q)$, respectively.

of space-time, and $\mu$ the 't Hooft mass; also, we will use roman letters to label Feynman diagrams computed in the $R_{\xi}$ gauge, and roman letters with hats when the same diagram is computed in the BFM gauge. For the perturbative analysis of this section, we will employ the scalar self-energy, $\Sigma$, related to the inverse scalar propagator by

$$
S^{-1}(q)=q^{2}-m^{2}+i \Sigma(q)
$$

and the same relation applies for $\widehat{S}^{-1}$ and $\widehat{\Sigma}$. In addition, we will use $S_{0}^{-1}(q)=q^{2}-m^{2}$.

\section{A. One-loop case}

The one-loop case is particularly simple. In fact, recall that we are working in the Feynman gauge; then, since graph (b) of Fig 2 can not possibly provide any pinching momenta, while graph (c) is zero in perturbation theory [due to Eq.(3.8) below], one needs to concentrate only on diagram (a). Then, by applying to the latter the decomposition described in Eq.(3.4), one should be able to generate graph $(\widehat{a})$, together with the rest of the terms appearing in the one-loop version of the BQI of Eq.(2.39), namely

$$
\Sigma^{(1)}(q)=\widehat{\Sigma}^{(1)}(q)-2 G^{(1)}(q) S_{0}^{-1}(q)
$$

Notice that, in the one loop case, the symbol [...] appearing in Eq.(3.4) is given by the expression $-i g_{\mu \nu} S_{0}(q+k) / k^{2}$. In what follows we will denote symbolically the application of Eq. (3.4) on (a) as

$$
(\mathrm{a})=(\mathrm{a})^{\mathrm{FF}}+(\mathrm{a})^{\mathrm{P} 0}+(\mathrm{a})^{0 \mathrm{P}}-(\mathrm{a})^{\mathrm{PP}} .
$$

The notation introduced above will be used extensively in the rest of the paper. 
The term (a) ${ }^{\mathrm{FF}}$ on the RHS of (3.7) represents already graph $(\widehat{\mathrm{a}})$ of Fig.2. $k$ ) coincides (by construction) with the tree-level $\widehat{\phi} \phi^{\dagger} A$ vertex. Then, using the tree-level version of the all-order WI given in Eq.(2.27), it is elementary to demonstrate that the second and third term on the RHS of (3.7) give each rise to a term $S_{0}^{-1}(q) G^{(1)}(q)$, with $G^{(1)}$ the one-loop version of Eq.(2.40). The last term, $(\mathrm{a})^{\mathrm{PP}}$, gives rise to a seagull-like graph in which the four scalar vertex is proportional to the gauge coupling $g^{2}$; it is this latter term combined with diagram (b) that will give rise to the characteristic BFM vertex $\phi^{\dagger} \phi \widehat{\phi}^{\dagger} \widehat{\phi} \propto\left(\lambda-g^{2}\right)$ (see the Appendix for its exact Feynman rule) and therefore to the diagram $(\widehat{b})$.

We end by observing that in carrying out the construction above we have used the dimensional regularization result $\int[d k] / k^{2}=0$, a special case of the more general formula

$$
\int \frac{[d k]}{k^{2}} \ln ^{N}\left(k^{2}\right)=0, \quad N=0,1,2, \ldots
$$

which guarantees the masslessness of the photon to all orders in perturbation theory [and that graph (c) is zero as well].

\section{B. Two-loop case}

The two-loop case is of course more involved; in fact, it has sufficient level of complexity to capture all central issues one needs to address for the all-order perturbative construction, as well as the generalization at the level of the SDEs, to be presented in the next sections.

As in the one-loop case, the idea is again to start out with the graphs defining the conventional two-loop scalar self-energy $\Sigma^{(2)}$, and to generate, via the application of the PT rules, the diagrams of the corresponding two-loop BFM self-energy $\widehat{\Sigma}^{(2)}$ together with all additional terms enforcing the BQI of (2.39) at two loops. In particular, the two-loop version of Eq.(2.39) is given by

$$
\Sigma^{(2)}(q)=\widehat{\Sigma}^{(2)}(q)-2 G^{(1)}(q) \Sigma^{(1)}(q)-2 G^{(2)}(q) S_{0}^{-1}(q)-\left[G^{(1)}(q)\right]^{2} S_{0}^{-1}(q) .
$$

Before entering into the details, we report the form of the all-order photon propagator, $\Delta_{\mu \nu}(k)$, in the Feynman gauge. We have

$$
\Delta_{\mu \nu}(k)=-i\left[\Delta\left(k^{2}\right) P_{\mu \nu}(k)+\frac{k_{\mu} k_{\nu}}{k^{4}}\right], \quad \Delta\left(k^{2}\right)=\frac{1}{k^{2}+i \Pi\left(k^{2}\right)},
$$

where $P_{\mu \nu}(k)=g_{\mu \nu}-k_{\mu} k_{\nu} / k^{2}$ denotes the dimensionless projection operator, and $\Pi_{\mu \nu}(k)=\Pi\left(k^{2}\right) P_{\mu \nu}(k)$ is the transverse vacuum polarization. 

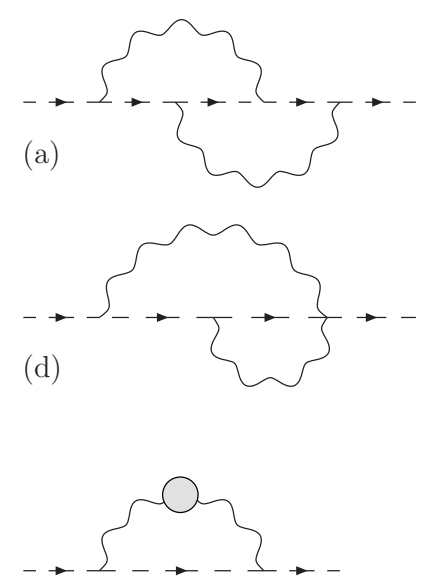

(g)

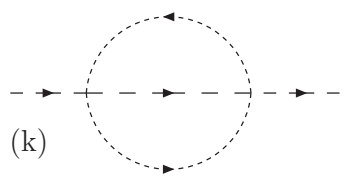

(b)

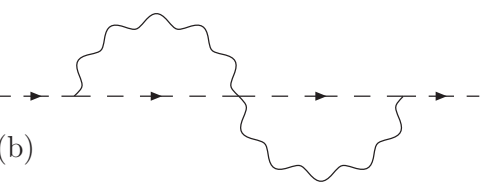

(e)
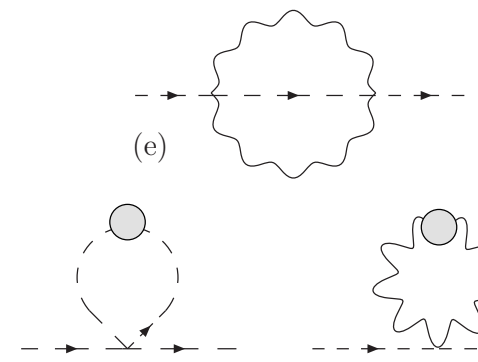

(h)

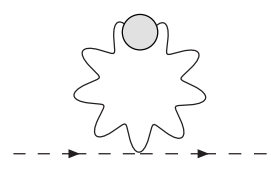

(i)
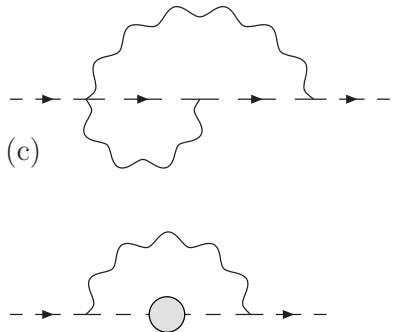

(f)

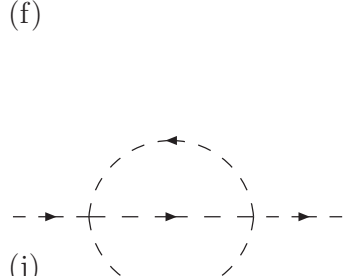

(j)

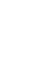

(1)

FIG. 3: The two-loop Feynman diagrams contributing to $\Sigma^{(2)}$ and $\widehat{\Sigma}^{(2)}$ : the topologies (a)-(j), evaluated using the corresponding set of Feynman rules, contribute to both, whereas $(\mathrm{k})$ only to the latter. Gray blobs represent the one-loop scalar and photon self-energy

The two-loop PT construction proceeds then as follows.

(i) $\Sigma^{(2)}$ is given by the sum of the one-particle irreducible (1PI) diagrams (a)-(j), shown in Fig [3; , on the other hand, $\widehat{\Sigma}^{(2)}$ is given by the sum (a)-(k) (in each case one would be of course using the corresponding set of Feynman rules). Notice in particular that diagram $(\mathrm{k})$ is due to the characteristic BFM vertex $\widehat{\phi} \phi^{\dagger} \bar{c} c$, shown in the Appendix.

(ii) Diagrams (a)-(j) of $\Sigma^{(2)}$ may be classified into three categories, according to the number of external $\phi^{\dagger} \phi A$ vertices they contain: type A diagrams with two such vertices - graphs (a), (b), (f), and (g); type B diagrams with one such vertex - graphs (c) and (d); type C diagrams with no such vertex - graphs (e), (h) (i) and (j). Then, to the type A diagrams one applies the rearrangement given in Eq.(3.4), whereas for the type B one simply carries out the PT splitting of Eq.(3.2) to their single external vertex. Finally, type $\mathrm{C}$ diagrams remain unchanged, as they do not contain any pinching momentum.

(iii) In type A graphs, the terms containing $\Gamma^{\mathrm{F}}[\ldots] \Gamma^{\mathrm{F}}$ give rise to the corresponding $\mathrm{BFM}$ 
diagrams, to be denoted by $(\widehat{\mathrm{a}}),(\widehat{\mathrm{b}}),(\widehat{\mathrm{f}})$, and $(\widehat{\mathrm{g}})$. Similarly, the terms containing $\Gamma^{\mathrm{F}}$ in type B graphs generate the corresponding BFM diagrams $(\widehat{c})$ and $(\widehat{d})$. Thus,

$$
\begin{aligned}
(\mathrm{x})^{\mathrm{FF}}=(\widehat{\mathrm{x}}) & \mathrm{x}=\mathrm{a}, \mathrm{b}, \mathrm{f}, \mathrm{g} \\
(\mathrm{y})^{\mathrm{F}}=(\widehat{\mathrm{y}}) & \mathrm{y}=\mathrm{c}, \mathrm{d}
\end{aligned}
$$

Notice that, due to the transversality of the photon self-energy, $k^{\mu} \Pi_{\mu \nu}^{(1)}(k)=0$, diagram (g) gives no further contributions, i.e. it has been converted into the corresponding diagram $(\widehat{\mathrm{g}})$ for free. As for diagram (f), using the tree-level version of Eq.(2.27), we have:

$$
\begin{aligned}
(\mathrm{f})^{\mathrm{P} 0}+(\mathrm{f})^{0 \mathrm{P}} & =2 g^{2} \int \frac{[d k]}{k^{2}} S_{0}(k+q) \Sigma^{(1)}(k+q)-2 G^{(2)}(q) S_{0}^{-1}(q) \\
(\mathrm{f})^{\mathrm{PP}} & =-g^{2} \int[d k] \Sigma^{(1)}(k) .
\end{aligned}
$$

(iv) We continue with the evaluation of the pinching parts of the remaining graphs considered in (iii). Combining the two type A graphs (a) and (b) with the two type B ones, we may organize the various contributions such that the two $\Gamma^{\mathrm{P}}$ are each acting on the full one-loop $\phi^{\dagger} \phi A$ vertex $\Gamma_{\mu}^{(1)}$, thus triggering the one-loop version of the WI of Eq.(2.27). Thus, the $\Gamma^{\mathrm{P}}$ on the left hand side (LHS) of diagrams (a), (b), and (d), will act on $\Gamma_{\mu}^{(1)}$, and exactly the same will happen with the $\Gamma^{\mathrm{P}}$ on the right hand side (RHS) of diagrams (a), (b), and (c). Specifically,

$$
\begin{aligned}
2\left[(\mathrm{a})^{\mathrm{P} 0}+(\mathrm{b})^{\mathrm{P} 0}+(\mathrm{d})^{\mathrm{P}}\right] & =2 g \int \frac{[d k]}{k^{2}} S_{0}(k+q) k^{\mu} \Gamma_{\mu}(-q, k+q) \\
& =-2 g^{2} \int \frac{[d k]}{k^{2}} S_{0}(k+q) \Sigma^{(1)}(k+q)-2 G^{(1)}(q) \Sigma^{(1)}(q),
\end{aligned}
$$

where the multiplicative factor of 2 accounts for the equal contribution from the symmetric combination $(\mathrm{a})^{\mathrm{OP}}+(\mathrm{b})^{\mathrm{OP}}+(\mathrm{c})^{\mathrm{P}}$. Finally, it is straightforward to demonstrate that

$$
(\mathrm{a})^{\mathrm{PP}}+(\mathrm{b})^{\mathrm{PP}}=-(\mathrm{k})+\left[G^{(1)}(q)\right]^{2} S_{0}^{-1}(q) .
$$

Evidently, diagram (k), originating from the special BFM ghost sector, has been generated dynamically from the rearrangement of diagrams evaluated with Feynman rules that do not involve ghost interactions. [To get the signs to work out, remember the 
minus sign in front of the $\Gamma^{\mathrm{P}}[\ldots] \Gamma^{\mathrm{P}}$ term, and the extra minus sign in $(\mathrm{k})$ due to the ghost loop.]

(v) Finally, taking into account the cancellation of the first terms on the RHS of Eqs.(3.12) and (3.13), we conclude that all diagrams contributing to $\widehat{\Sigma}^{(2)}$ have been generated by pinching internally $\Sigma^{(2)}$, together with all terms on the RHS of (3.9); this concludes the two-loop construction of the PT scalar self-energy.

As has been explained in detail in the literature (for the more complicated non-Abelian case) [40], all terms in Eq.(3.9) containing the auxiliary function $G$ will eventually cancel exactly in an $S$-matrix element (or other physical observable) against similar contributions coming from the conversion of the two-loop conventional vertex $\Gamma_{\mu}^{(2)}$ to the PT-BFM vertex $\widehat{\Gamma}_{\mu}^{(2)}$, [viz. Eq.(2.43)], together with analogous terms originating from the conversion of the $1 \mathrm{PR}$ strings (i.e. products of conventional one-loop vertices and self-energies) into PT strings, e.g., 1PR strings containing instead products of one-loop PT vertices and selfenergies.

\section{PINCHING SCHWINGER-DYSON EQUATIONS}

We now enter into the main issue of this article, namely how to carry out the PT construction at the level of SDE. In this section we will present a general qualitative discussion of the main questions involved, the strategy that will be employed, and the field-theoretic ingredients necessary for its implementation. The actual detailed construction will be presented in Section VI.

The SDE may be derived following a diagrammatic analysis in the spirit of [42], or formally from the generating functional of the theory, as shown, for example, in [43]. For the case of scalar QED, the SDE for the scalar propagator $S$ is shown in Fig 4, it essentially amounts to dressing with vertex and self-energy corrections all skeleton graphs contributing to $S$. In scalar QED the skeleton graphs of the scalar (and photon) propagator are exhausted at two loops; this is equivalent to saying that, with the elementary vertices at hand, any higher order graph is bound to be a radiative correction (propagator or vertex "dressing") to the one- and two-loop graphs. That this is so may be verified by direct diagrammatic analysis, analogous to the one presented in [42] for the case of spinor QED. In general, the 
number of skeleton graphs depends on the type of elementary vertices characterizing the theory. Thus, in spinor QED the fermion and electron self-energies have a single skeleton graph, the analogue of graph (4a); the same is true for a $g \phi^{3}$ theory. In both cases the reason is that there exists only one fundamental interaction vertex. Instead, in the case of a $g \phi^{3}+\lambda \phi^{4}$ theory, of scalar QED, and of QCD, due to the presence of interaction vertices involving four fields, two-loop skeleton graphs exist; for example, no radiative correction to graph (2a) could possibly give rise to the graphs (3c) and (3e). Clearly, field theories with elementary vertices involving more than four fields have self-energy skeleton graphs beyond two loops.

There are three fundamental (fully dressed, all-order) vertices appearing in the SDE of $S$, corresponding to the couplings $\partial_{\mu} \phi^{\dagger} \phi A^{\mu}, \phi^{\dagger} \phi A^{\mu} A_{\mu}$, and $\phi^{\dagger} \phi \phi^{\dagger} \phi$, to be denoted by $\Gamma_{\mu}, \Gamma_{\mu \nu}$, and $\Gamma$, respectively. The corresponding SDEs are shown in Fig.5. Their general structure may be described as follows: A vertex-leg is singled out (in our case the scalar leg carrying momentum $q$ ), and all possible tree-level vertices involving this field (leg) are written down. Then, the fields exiting from these tree-level vertices are either $(i)$ all connected with the remaining vertex-legs through appropriate multi-particle kernels, or (ii) one of them is directly identified with one of the vertex-legs, whereas the rest is connected to the remaining vertex-legs through an appropriate kernel or full vertex. The various kernels involved (to be denoted in what follows as $\mathcal{K}_{\mu \nu}$ and $\mathcal{K}_{\mu \nu \rho}$ ) are connected, and, in addition, 1PI with respect to cuts involving only a physical momentum; this is tantamount to saying that these kernels do not contain graphs that could become disconnected by cutting a single line that is irrigated exclusively by one of the external, ("physical" as opposed to "virtual") momenta entering into the vertex. It is important to emphasize that there is a finite number of distinct $n$-particle kernels appearing in the SDE for the vertices of Fig 5 . Specifically, the SDE of a vertex with $m$-fields ( $m=3,4$, in our case) will involve all kernels with $n \leq m+2$. To see with an example why this must be so, consider the SDE of $\Gamma_{\mu}$ in Fig.5, and let us add one additional leg to the 5-particle kernel appearing either in (e) or in (h). Since the number of external legs is fixed, this extra leg must be attached to the rest of the diagram through an internal elementary vertex. The resulting graph, however, will be nothing but a radiative correction to one of the graphs containing the kernels with $n \leq 5$; therefore, its inclusion would constitute overcounting. Notice finally that we do not consider the SDE for the photon propagator, because in scalar QED it remains completely inert in the PT 


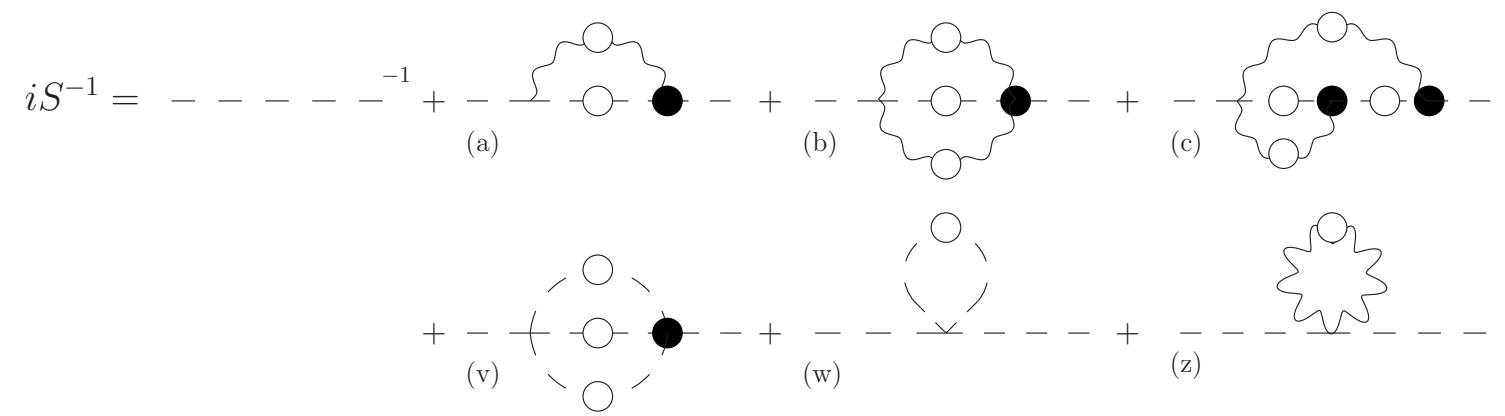

FIG. 4: Schwinger-Dyson equations for the scalar self-energy $i S^{-1}(q)$. The charge flow is not shown. Here black blobs represent 1 PI Green's functions $\left(\Gamma_{\mu}, \Gamma_{\mu \nu}\right.$ and $\left.\Gamma\right)$, and white blobs connected Green's functions $\left(\Delta_{\mu \nu}\right.$ and $\left.S\right)$.

rearrangement (the most direct way to see this is by noticing that all couplings involving a conventional photon coincide with those containing a background photon).

The key observations that allow for the extension of the PT algorithm at the level of the SDE of the theory are then the following.

(i) In order to carry out the PT construction for the SDE at hand, it is important to first identify the origin of the pinching momenta (i.e., type A and B diagrams), and then the structures (vertices, kernels, etc) these momenta will be acting upon. Let us focus for concreteness on the SDE for the scalar propagator $S$. To determine the pinching momenta, we apply the same criterion as in the perturbative case, namely we carry out the PT decomposition to the "external" vertices. Looking at the diagrams of Fig, 4 , it is clear that the bare vertex on the very left of diagram (a) should be decomposed according to Eq.(3.2); what is less clear perhaps is how to implement the subsequent splitting described in Eq.(3.4), or in other words, identify the second external vertex to be decomposed. The perturbative examples studied in the previous section suggest that the second external vertex resides inside the black blob denoting the full trilinear vertex $\Gamma_{\mu}$ in (a); thus, in order to implement Eq.(3.4) one must "unwrap" $\Gamma_{\mu}$. This

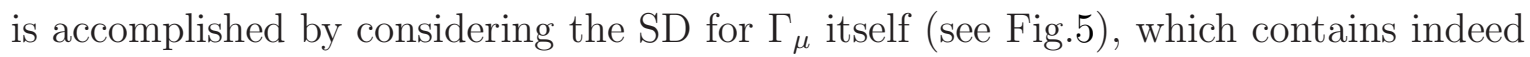
tree-level vertices $\Gamma_{\mu}^{(0)}$, on its RHS (the first two terms). Then, one must think of the RHS of this latter SDE as having been inserted in (a), instead of $\Gamma_{\mu}$, and carry out Eq.(3.2) on the tree-level vertices $\Gamma_{\mu}^{(0)}$ now appearing explicitly on the right of (a), see, e.g., Fig:7. 


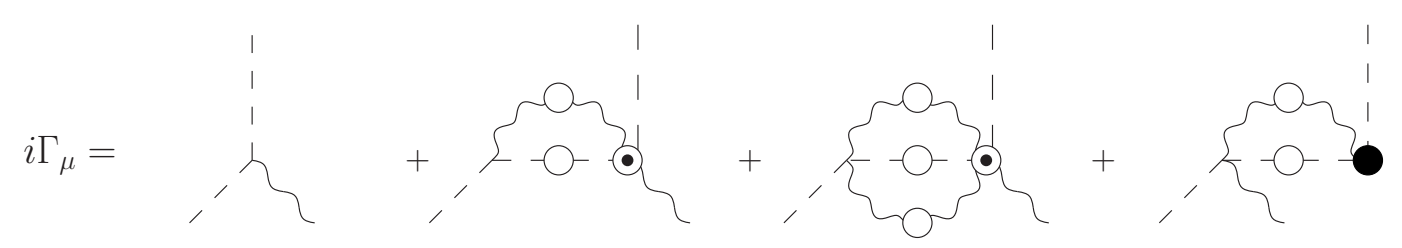

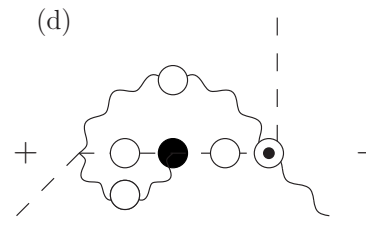

(g)

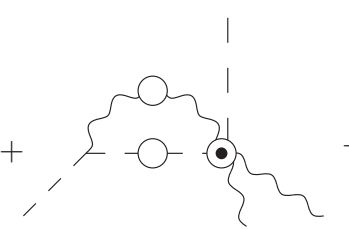

(i)

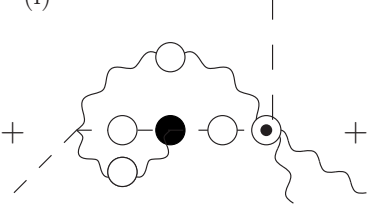

(l)

(f)

(e)

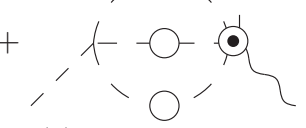

(h)

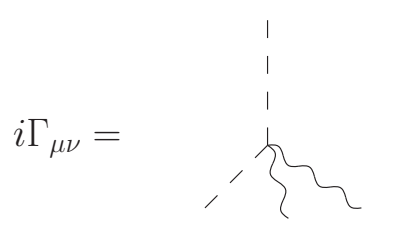

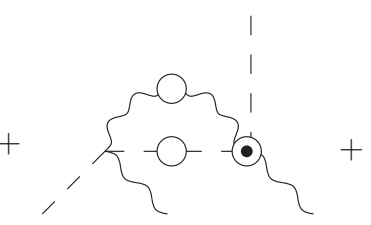

$(\mathrm{j})$

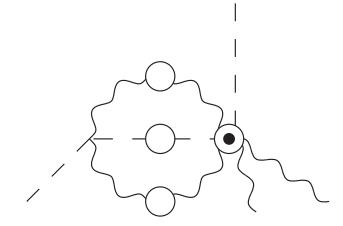

(k)

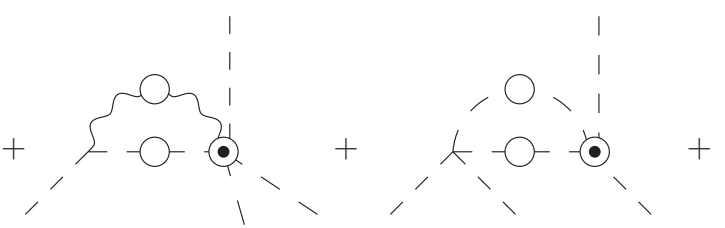

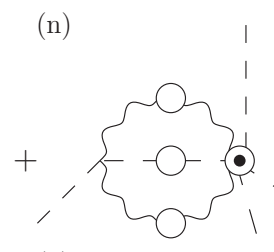

(q) (o)

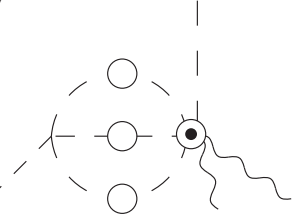

(m)

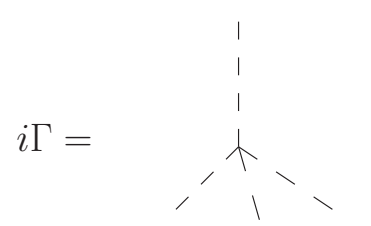

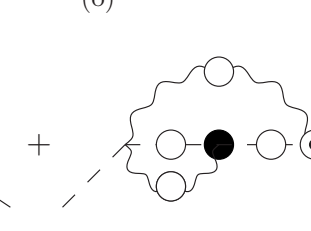

(r)

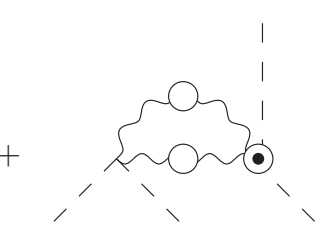

(p)

FIG. 5: Schwinger-Dyson equations for the three fundamental vertices, $i \Gamma_{\mu}\left(p_{1}, p_{2}\right), i \Gamma_{\mu \nu}\left(k, p_{1}, p_{2}\right)$ and $i \Gamma\left(p_{1}, p_{2}, p_{3}\right)$. As before, black blobs represent 1 PI Green's functions $\left(\Gamma_{\mu}\right)$, white blobs connected Green's functions $\left(\Delta_{\mu \nu}\right.$ and $\left.S\right)$, and white blobs with a black center denote the various kernels.

(ii) After having settled the question of how to identify type A diagrams and how to carry out the implementation of Eq.(3.4) in the presence of a full trilinear vertex, the next question is what the result of this operation will be, and in particular the contributions of the terms $\Gamma_{\mu}^{\mathrm{P}}[\ldots] \Gamma_{\nu}^{(0)}, \Gamma_{\mu}^{(0)}[\ldots] \Gamma_{\nu}^{\mathrm{P}}$, and $\Gamma_{\mu}^{\mathrm{P}}[\ldots] \Gamma_{\nu}^{\mathrm{P}}$. It is clear that the $\Gamma^{\mathrm{P}}$ originating from the tree-level vertex on the left of (a) will trigger directly the WI of Eq.(2.27), since it acts on a full $\Gamma_{\mu}$. The result of the action of the $\Gamma^{\mathrm{P}}$ coming from the 
other side is, however, less transparent. Of course, our perturbative experience tells us that $\Gamma_{\mu}^{\mathrm{P}}[\ldots] \Gamma_{\nu}^{(0)}$ and $\Gamma_{\mu}^{(0)}[\ldots] \Gamma_{\nu}^{\mathrm{P}}$ should give identical contributions; however, unlike the perturbative case where the symmetry of the situation is manifest, now one has to demonstrate that this is indeed the case. The way this is done is by noticing that, just as happened in the two-loop case where $(\mathrm{a})^{0 \mathrm{P}}$ was combined with $(\mathrm{b})^{\mathrm{OP}}$ and $(\mathrm{c})^{\mathrm{P}}$ to generate $\Gamma_{\mu}^{(1)}$, now one has to consider the analogous contributions from graphs (b), (c), and (w) of Fig, 4. Specifically, one must carry out the PT decomposition on the corresponding full vertices (black "blobs"), appearing on the very right of these graphs; this is accomplished again by unwrapping them, invoking their own SDE's, and carrying out Eq.(3.2) on the tree-level trilinear vertices appearing on their RHS. The end-result of this will be that the $\Gamma^{\mathrm{P}}$ coming from the right will be acting on a set of diagrams that will be precisely the RHS of the SDE for $\Gamma_{\mu}$; thus, the WI of Eq.(2.27) will be triggered again, as expected.

(iii) Next we turn to the term $\Gamma_{\mu}^{\mathrm{P}}[\ldots] \Gamma_{\nu}^{\mathrm{P}}$. It should be clear from the two-loop construction, that what is contained in [...] of (a) and (b) in Fig.3 is nothing but the tree-level 1PI kernel containing two scalars and two photons, i.e., $\mathcal{K}_{\mu \nu}^{(0)}$. So, what one is actually computing at two-loop in order to arrive at Eq.(3.14) is the tree-level WI for $\mathcal{K}_{\mu \nu}$. This observation persists at the level of the propagator SDE: to determine $\Gamma_{\mu}^{\mathrm{P}}[\ldots] \Gamma_{\nu}^{\mathrm{P}}$ one must find the result of fully contracting $\mathcal{K}_{\mu \nu}$ by the corresponding momenta carried by the two photons entering.

(iv) Turning to the SD equations for the vertices, let us first observe that the PT procedure can be implemented by simply carrying out the PT decomposition to the corresponding vertices $\Gamma_{\mu}^{(0)}$ appearing on the corresponding RHS. Thus, for the case of $\Gamma_{\mu}$ one must decompose the $\Gamma_{\mu}^{(0)}$ appearing in graph (d), and determine the action of the longitudinal momentum on the kernel $\mathcal{K}_{\mu \nu}$. For the case of $\Gamma_{\mu \nu}$ one must do the same in graph (i), and thus determine the action of the longitudinal momentum coming from $\Gamma_{\mu}^{\mathrm{P}}$ on the five-particle (three photons and two scalars) kernel $\mathcal{K}_{\mu \nu \rho}$.

$(v)$ Let us also emphasize that, for the purpose of pinching the propagator SDE alone, one does not need the WIs for the multi-particle kernels appearing in the various SDEs, other than $\mathcal{K}_{\mu \nu}$. Indeed, as has been outlined above, when the relevant contributions from the vertices are inserted into the SDE of $S$, and are appropriately combined with 
other graphs, the pinching momentum acts finally on a full $\Gamma_{\mu}$, triggering its known WI. The need for the WI satisfied by $\mathcal{K}_{\mu \nu \rho}$, etc. arises only if one decides to pinch in addition the SDEs for $\Gamma_{\mu}, \Gamma_{\mu \nu}$, and $\Gamma$. In Section VI we will pinch the SDE for $\Gamma_{\mu}$ and $\Gamma_{\mu \nu}$, but will skip the case of the four-scalar vertex $\Gamma$; the latter is straightforward but tedious, and presents limited conceptual or practical interest. Thus, the only WIs needed for our purposes are those for the kernels $\mathcal{K}_{\mu \nu}$ and $\mathcal{K}_{\mu \nu \rho}$.

Summarizing, we have seen that the PT construction can be carried out at the level of the SDE, when appropriate adjustments to the perturbative methodology are implemented. In particular, in the construction of the PT scalar self-energy $S(q)$, in addition to its own SDE, one must simultaneously consider the SDE for the full vertices involved, manipulating them appropriately. Furthermore, it has become clear that one needs to derive closed expressions for the all-order WI satisfied by 1PI multi-particle kernels. This question will be addressed in detail in the next section.

\section{WARD IDENTITIES FOR KERNELS}

In this section we will derive the WI needed for carrying out the PT construction for the SDEs of $S, \Gamma_{\mu}$, and $\Gamma_{\mu \nu}$. As discussed above, this would require the WI for the kernels $\mathcal{K}_{\mu \nu}$ and $\mathcal{K}_{\mu \nu \rho}$ appearing in them. Of course, in the context of the Abelian theory that we consider, the tree-level WIs ought to generalize naively to all orders, with no ghost contributions. Thus, as a short-cut, one could simply derive the tree-level results and postulate their validity to all orders. Instead we will derive the relevant all-order WIs formally, not only for completeness, but also in order to establish the necessary theoretical framework for addressing the same question in the more complicated case of non-Abelian theories.

The main subtlety involved in this treatment stems from the fact that the standard techniques furnish WIs for the connected kernels instead of the 1PI ones (in the sense described in the previous section), i.e., for $\mathcal{C}_{\mu \nu}$ instead of the $1 \mathrm{PI} \mathcal{K}_{\mu \nu}$ appearing in the SDEs. Therefore, in order to obtain the desired results, one must properly account for the 1PR terms, and subtract their contributions from the WIs derived for the connected kernels $\mathcal{C}$.

In order to determine formally the all-order WI satisfied by $\mathcal{C}$ kernels, we proceed as described in [43]. We start by considering the Lagrangian of Eq.(2.2); as a consequence of 
its invariance under the gauge transformations

$$
\phi(x) \rightarrow e^{i \alpha(x)} \phi(x), \quad \phi^{\dagger}(x) \rightarrow e^{-i \alpha(x)} \phi^{\dagger}(x), \quad A_{\mu} \rightarrow A_{\mu}+\frac{1}{g} \partial_{\mu} \alpha(x),
$$

one has the conservation of the current

$$
J_{\rho}(x)=i: \phi^{\dagger}(x) \overleftrightarrow{\partial_{\rho}} \phi(x):+2 g: A_{\rho}(x) \phi^{\dagger}(x) \phi(x):, \quad \partial^{\rho} J_{\rho}(x)=0
$$

Then, one can derive WIs relating Green's functions involving a single current operator and an arbitrary number of scalar and photon fields, as a result of current conservation and the fact that Green's functions are expressed as time-ordered products in Minkowski space. Specifically, one has that

$$
\begin{aligned}
& \partial_{x}^{\rho}\left\langle 0\left|T J_{\rho}(x) \prod_{i=1}^{n} A_{\rho_{i}}\left(z_{i}\right) \prod_{j=1}^{m} \phi^{\dagger}\left(y_{j}\right) \phi\left(x_{j}\right)\right| 0\right\rangle \\
= & \sum_{k=1}^{n}\left\langle 0\left|T\left[J_{0}(x), A_{\rho_{k}}\left(z_{k}\right)\right] \delta\left(x^{0}-z_{k}^{0}\right) \prod_{i=1, i \neq k}^{n} A_{\rho_{i}}\left(z_{i}\right) \prod_{j=1}^{m} \phi^{\dagger}\left(y_{j}\right) \phi\left(x_{j}\right)\right| 0\right\rangle \\
+ & \sum_{k=1}^{m}\langle 0| T \prod_{i=1}^{n} A_{\rho_{i}}\left(z_{i}\right)\left\{\left[J_{0}(x), \phi^{\dagger}\left(y_{k}\right)\right] \delta\left(x^{0}-y_{k}^{0}\right) \phi\left(x_{k}\right)+\phi^{\dagger}\left(y_{k}\right)\left[J_{0}(x), \phi\left(x_{k}\right)\right] \delta\left(x^{0}-x_{k}^{0}\right)\right\} \times \\
\times & \prod_{j=1, j \neq k}^{m} \phi^{\dagger}\left(y_{j}\right) \phi\left(x_{j}\right)|0\rangle,
\end{aligned}
$$

where the term containing $\partial^{\rho} J_{\rho}$ have been set to zero. On the other hand, canonical equaltime commutation relations (which ensure charge conservation) imply

$$
\begin{aligned}
{\left[J_{0}(x), \phi\left(x^{\prime}\right)\right] \delta\left(x^{0}-x^{\prime 0}\right) } & =g \phi(x) \delta^{4}\left(x-x^{\prime}\right), \\
{\left[J_{0}(x), \phi^{\dagger}\left(x^{\prime}\right)\right] \delta\left(x^{0}-x^{\prime 0}\right) } & =-g \phi^{\dagger}(x) \delta^{4}\left(x-x^{\prime}\right), \\
{\left[J_{0}(x), A_{\rho}\left(x^{\prime}\right)\right] \delta\left(x^{0}-x^{\prime 0}\right) } & =0 .
\end{aligned}
$$

We arrive then at the following general WI

$$
\begin{aligned}
& \partial_{x}^{\rho}\left\langle 0\left|T J_{\rho}(x) \prod_{i=1}^{n} A_{\rho_{i}}\left(z_{i}\right) \prod_{j=1}^{m} \phi^{\dagger}\left(y_{j}\right) \phi\left(x_{j}\right)\right| 0\right\rangle \\
= & -g\left\langle 0\left|T \prod_{i=1}^{n} A_{\rho_{i}}\left(z_{i}\right) \prod_{j=1}^{m} \phi^{\dagger}\left(y_{j}\right) \phi\left(x_{j}\right)\right| 0\right\rangle \sum_{k=1}^{m}\left[\delta^{4}\left(x-y_{k}\right)-\delta^{4}\left(x-x_{k}\right)\right] .
\end{aligned}
$$

This identity constitutes our starting point for deriving the WIs satisfied by the SD kernels $\mathcal{K}_{\mu \nu}$ and $\mathcal{K}_{\mu \nu \rho}$. 

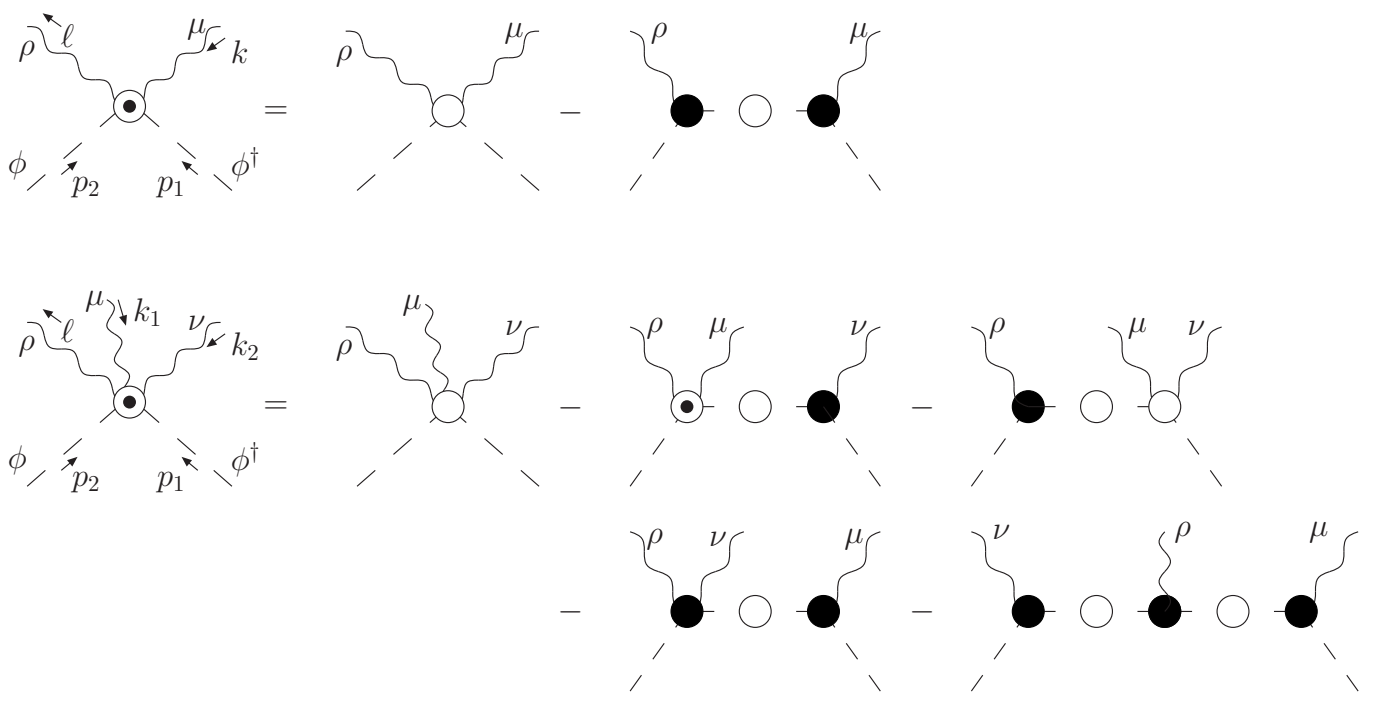

FIG. 6: The four- and five-particle kernels $\mathcal{K}_{\rho \mu}\left(k, p_{1}, p_{2}\right)$ and $\mathcal{K}_{\rho \mu \nu}\left(k_{1}, k_{2}, p_{1}, p_{2}\right)$ appearing in the $S D E s$, and their relations with the corresponding amputated connected Green's function $\mathcal{C}_{\rho \mu}$ and $\mathcal{C}_{\rho \mu \nu}$ and 1 PI Green's functions $\Gamma_{\mu}$ and $\Gamma_{\mu \nu}$.

\section{A. Four particle kernel}

The first case of interest for us is when $n=2$ and $m=1$, i.e., the photon-photon-scalarscalar scattering kernel, defined as

$$
\begin{aligned}
& \int d^{4} x d^{4} x_{1} d^{4} y_{1} d^{4} z_{1} e^{i\left(p_{1} \cdot y_{1}+p_{2} \cdot x_{1}+k \cdot z_{1}-\ell \cdot x\right)}\left\langle 0\left|T J^{\rho}(x) A_{\beta}\left(z_{1}\right) \phi^{\dagger}\left(y_{1}\right) \phi\left(x_{1}\right)\right| 0\right\rangle i \Delta_{\rho \alpha}^{(0)}(\ell) \\
= & (2 \pi)^{4} \delta^{4}\left(k+p_{1}+p_{2}-\ell\right) \widetilde{\mathcal{C}}_{\alpha \beta}\left(k, p_{1}, p_{2}\right) .
\end{aligned}
$$

Contraction with $\ell^{\alpha}$ gives

$$
\begin{aligned}
& (2 \pi)^{4} \delta^{4}\left(k+p_{1}+p_{2}-\ell\right) \ell^{\alpha} \widetilde{\mathcal{C}}_{\alpha \beta}\left(k, p_{1}, p_{2}\right) \\
= & -\frac{1}{\ell^{2}} \int d^{4} x d^{4} x_{1} d^{4} y_{1} d^{4} z_{1} e^{i\left(p_{1} \cdot y_{1}+p_{2} \cdot x_{1}+k \cdot z_{1}-\ell \cdot x\right)} \partial_{\rho}^{x}\left\langle 0\left|T J^{\rho}(x) A_{\beta}\left(z_{1}\right) \phi^{\dagger}\left(y_{1}\right) \phi\left(x_{1}\right)\right| 0\right\rangle,
\end{aligned}
$$

and therefore, using Eq.(5.5) with $n=2$ and $m=1$, we get

$$
\begin{aligned}
& (2 \pi)^{4} \delta^{4}\left(k+p_{1}+p_{2}-\ell\right) \ell^{\mu} \widetilde{\mathcal{C}}_{\alpha \beta}\left(k, p_{1}, p_{2}\right) \\
= & \frac{1}{\ell^{2}} \int d^{4} x_{1} d^{4} y_{1} d^{4} z_{1} e^{i\left[\left(p_{1}-\ell\right) \cdot y_{1}+p_{2} \cdot x_{1}+k \cdot z_{1}\right]} g\left\langle 0\left|T A_{\beta}\left(z_{1}\right) \phi^{\dagger}\left(y_{1}\right) \phi\left(x_{1}\right)\right| 0\right\rangle \\
- & \frac{1}{\ell^{2}} \int d^{4} x_{1} d^{4} y_{1} d^{4} z_{1} e^{i\left[\left(p_{2}-\ell\right) \cdot x_{1}+p_{1} \cdot y_{1}+k \cdot z_{1}\right]} g\left\langle 0\left|T A_{\beta}\left(z_{1}\right) \phi^{\dagger}\left(y_{1}\right) \phi\left(x_{1}\right)\right| 0\right\rangle .
\end{aligned}
$$

Finally, defining the photon-scalar-scalar kernel $\widetilde{\mathcal{C}}_{\beta}$ as

$$
-i(2 \pi)^{4} \delta^{4}\left(p_{1}+p_{2}-k\right) \widetilde{\mathcal{C}}_{\beta}\left(p_{1}, p_{2}\right)
$$




$$
=\int d^{4} x d^{4} x_{1} d^{4} y_{1} e^{i\left(p_{1} \cdot y_{1}+p_{2} \cdot x_{1}-k \cdot x\right)}\left\langle 0\left|T A_{\beta}(x) \phi^{\dagger}\left(y_{1}\right) \phi\left(x_{1}\right)\right| 0\right\rangle
$$

and introducing the amputated kernels

$$
\begin{aligned}
& \widetilde{\mathcal{C}}_{\alpha \beta}\left(k, p_{1}, p_{2}\right)=i \Delta_{\alpha \rho}(\ell) i \Delta_{\beta \mu}(k) i S\left(p_{1}\right) i S\left(p_{2}\right) \mathcal{C}^{\rho \mu}\left(k, p_{1}, p_{2}\right), \\
& \widetilde{\mathcal{C}}_{\beta}\left(p_{2}, p_{1}\right)=i \Delta_{\beta \mu}(k) i S\left(p_{1}\right) i S\left(p_{2}\right) \Gamma^{\mu}\left(p_{2}, p_{1}\right),
\end{aligned}
$$

we obtain the required WI,

$$
\ell^{\rho} \mathcal{C}_{\rho \mu}\left(k, p_{1}, p_{2}\right)=g\left[S^{-1}\left(p_{1}\right) S\left(p_{1}-\ell\right) \Gamma_{\mu}\left(p_{1}-\ell, p_{2}\right)-S^{-1}\left(p_{2}-\ell\right) S\left(p_{2}\right) \Gamma_{\mu}\left(p_{1}, p_{2}-\ell\right)\right],
$$

or, making use of momentum conservation,

$$
\ell^{\rho} \mathcal{C}_{\rho \mu}\left(k, p_{1}, p_{2}\right)=g\left[S^{-1}\left(p_{1}\right) S\left(p_{2}+k\right) \Gamma_{\mu}\left(-k-p_{2}, p_{2}\right)-S^{-1}\left(p_{2}\right) S\left(p_{2}-\ell\right) \Gamma_{\mu}\left(p_{1},-k-p_{1}\right)\right]
$$

Notice that, in our $U(1)$ case, one could equally well contract with $\ell^{\rho}$ all the diagrams appearing in the decomposition of $\mathcal{C}$ shown in Fig, using the WI of Eq.(2.27) and Eq.(2.31); the result would be of course the same. The contraction of Eq.(5.12) with $k^{\mu}$ can now be easily evaluated using the WI of Eq.(2.27), and gives

$$
k^{\mu} \ell^{\rho} \mathcal{C}_{\rho \mu}\left(k, p_{1}, p_{2}\right)=g^{2}\left\{S^{-1}\left(p_{1}\right)+S^{-1}\left(p_{2}\right)-S^{-1}\left(p_{1}\right)\left[S\left(p_{2}+k\right)+S\left(p_{1}+k\right)\right] S^{-1}\left(p_{2}\right)\right\} .
$$

As explained in the general analysis carried out in the previous section, we will need the WIs satisfied by the kernel $\mathcal{K}_{\rho \mu}$, and not the ones for the connected Green's function $\mathcal{C}_{\rho \mu}$. These former WIs are however easily obtained, by making use of the relation (see Fig, 6)

$$
i \mathcal{K}_{\rho \mu}\left(k, p_{1}, p_{2}\right)=i \mathcal{C}_{\rho \mu}\left(k, p_{1}, p_{2}\right)-i \Gamma_{\rho}\left(\ell-p_{2}, p_{2}\right) i S\left(p_{2}-\ell\right) i \Gamma_{\mu}\left(p_{1}, p_{2}-\ell\right)
$$

Contracting with $\ell^{\rho}$ and $k^{\mu}$, and using Eqs.(5.12), (5.13) and (2.31), we then arrive at the desired WIs, which read

$$
\begin{aligned}
\ell^{\rho} \mathcal{K}_{\rho \mu}\left(k, p_{1}, p_{2}\right) & =g\left[\Gamma_{\mu}\left(-k-p_{2}, p_{2}\right) S^{-1}\left(p_{1}\right) S\left(k+p_{2}\right)-\Gamma_{\mu}\left(p_{1},-k-p_{1}\right)\right], \\
k^{\mu} \ell^{\mu} \mathcal{K}_{\rho \mu}\left(k, p_{1}, p_{2}\right) & =g^{2}\left[S^{-1}\left(k+p_{1}\right)-S^{-1}\left(p_{1}\right) S\left(k+p_{2}\right) S^{-1}\left(p_{2}\right)\right] .
\end{aligned}
$$




\section{B. Five particle kernel}

The second case of interest for our construction is the one where $n=3$ and $m=1$, i.e., the photon-photon-photon-scalar-scalar scattering kernel, defined as

$$
\begin{aligned}
& \int d^{4} x d^{4} x_{1} d^{4} y_{1} d^{4} z_{1} d^{4} z_{2} e^{i\left(p_{1} \cdot y_{1}+p_{2} \cdot x_{1}+k_{1} \cdot z_{1}+k_{2} \cdot z_{2}-\ell \cdot x\right)} \times \\
\times & \left\langle 0\left|T J^{\rho}(x) A_{\beta}\left(z_{1}\right) A_{\gamma}\left(z_{2}\right) \phi^{\dagger}\left(y_{1}\right) \phi\left(x_{1}\right)\right| 0\right\rangle i \Delta_{\rho \alpha}^{(0)}(\ell) \\
= & i(2 \pi)^{4} \delta^{4}\left(k_{1}+k_{2}+p_{1}+p_{2}-\ell\right) \widetilde{\mathcal{C}}_{\alpha \beta \gamma}\left(k_{1}, k_{2}, p_{1}, p_{2}\right) .
\end{aligned}
$$

Proceeding in exactly the same way as in the four-particle case, and introducing the amputated kernel

$$
\widetilde{\mathcal{C}}_{\alpha \beta \gamma}\left(k_{1}, k_{2}, p_{1}, p_{2}\right)=i \Delta_{\alpha \rho}(\ell) i \Delta_{\beta \mu}\left(k_{1}\right) i \Delta_{\gamma \nu}\left(k_{2}\right) i S\left(p_{1}\right) i S\left(p_{2}\right) \mathcal{C}^{\rho \mu \nu}\left(k_{1}, k_{2}, p_{1}, p_{2}\right),
$$

we obtain the WI

$$
\begin{aligned}
\ell^{\rho} \mathcal{C}_{\rho \mu \nu}\left(k_{1}, k_{2}, p_{1}, p_{2}\right) & =g\left[S^{-1}\left(p_{1}\right) S\left(p_{1}-\ell\right) \mathcal{C}_{\mu \nu}\left(k_{2}, p_{1}-\ell, p_{2}\right)\right. \\
& \left.-S^{-1}\left(p_{2}-\ell\right) S\left(p_{2}\right) \mathcal{C}_{\mu \nu}\left(k_{2}, p_{1}, p_{2}-\ell\right)\right]
\end{aligned}
$$

Once again, the connected kernel $\mathcal{C}_{\rho \mu \nu}$ is not the one that appears in the SDEs, being related to the latter through the equation (see Fig [6)

$$
\begin{aligned}
i \mathcal{K}_{\rho \mu \nu}\left(k_{1}, k_{2}, p_{1}, p_{2}\right) & =i \mathcal{C}_{\rho \mu \nu}\left(k_{1}, k_{2}, p_{1}, p_{2}\right)-i \Gamma_{\rho}\left(\ell-p_{2}, p_{2}\right) i S\left(p_{2}-\ell\right) i \mathcal{C}_{\mu \nu}\left(k_{2}, p_{1}, p_{2}-\ell\right) \\
& -i \mathcal{K}_{\rho \mu}\left(k_{1}, p_{1}+k_{2}, p_{2}\right) i S\left(p_{1}+k_{2}\right) i \Gamma_{\nu}\left(p_{1},-p_{1}-k_{2}\right) \\
& -i \Gamma_{\rho \nu}\left(k_{2}, p_{1}+k_{1}, p_{2}\right) i S\left(p_{1}+k_{1}\right) i \Gamma_{\mu}\left(p_{1},-p_{1}-k_{1}\right) \\
& -i \Gamma_{\nu}\left(-k_{2}-p_{2}, p_{2}\right) i S\left(p_{2}+k_{2}\right) i \Gamma_{\rho}\left(k_{1}+p_{1}, k_{2}+p_{2}\right) i S\left(p_{1}+k_{1}\right) \times \\
& \times i \Gamma_{\mu}\left(p_{1},-p_{1}-k_{1}\right) .
\end{aligned}
$$

Contracting with $\ell^{\rho}$, and making use of the WIs of Eqs.(5.12), (15.15), (15.13) and (2.31) we obtain, after a lengthy but straightforward calculation, the desired result

$$
\begin{aligned}
\ell^{\rho} \mathcal{K}_{\rho \mu \nu}\left(k_{1}, k_{2}, p_{1}, p_{2}\right) & =g S^{-1}\left(p_{1}\right) S\left(p_{1}-\ell\right) \mathcal{C}_{\mu \nu}\left(k_{2}, p_{1}-\ell, p_{2}\right)-g \Gamma_{\mu \nu}\left(k_{2}, p_{1},-k_{2}-k_{1}-p_{1}\right) \\
& +g \Gamma_{\mu}\left(-k_{1}-p_{2}, p_{2}\right) S\left(k_{1}+p_{2}\right) \Gamma_{\nu}\left(p_{1},-p_{1}-k_{2}\right) \\
& +g \Gamma_{\nu}\left(-k_{2}-p_{2}, p_{2}\right) S\left(k_{2}+p_{2}\right) \Gamma_{\mu}\left(p_{1},-p_{1}-k_{1}\right) .
\end{aligned}
$$



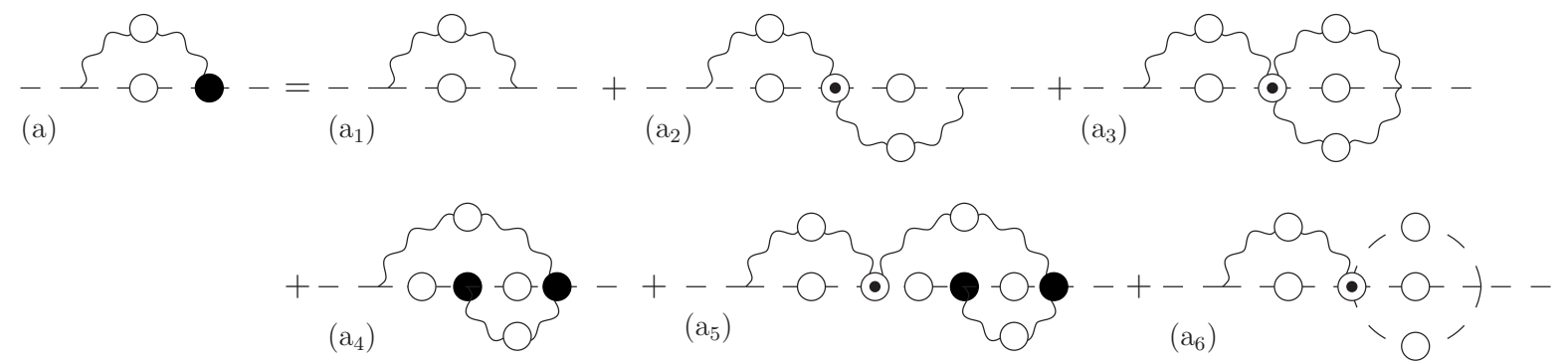

(b)
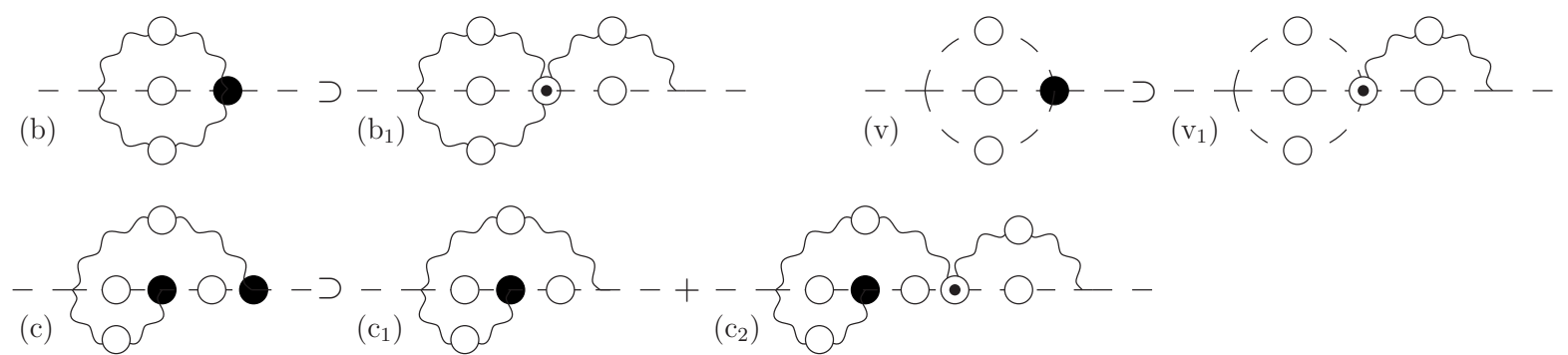

FIG. 7: Isolating from the SDE of the scalar self-energy all the terms providing PT amplitudes.

\section{PT GREEN'S FUNCTIONS FROM SCHWINGER-DYSON EQUATIONS}

In this section we will carry out in detail the PT construction at the level of the SDEs. Specifically, from the SDEs shown in Figs, 4 and 5, we will derive the PT Green's functions for the scalar propagator $S$, the trilinear vertex $\Gamma_{\mu}$, and the quadrilinear vertex $\Gamma_{\mu \nu}$.

\section{A. The scalar propagator}

As far as the SDE of the scalar propagator is concerned, the first step will be to isolate all the type A and type B diagrams, on which one could implement the characteristic PT decomposition of Eqs. (3.2) and (3.4). To this end, let us start with diagram (a) of Fig.4 and unwrap the full $\Gamma_{\mu}$ by means of its own SDE. The result is shown in the first two lines of Fig:7, clearly $\left(\mathrm{a}_{1}\right)$ and $\left(\mathrm{a}_{2}\right)$ are type A diagrams, while $\left(\mathrm{a}_{3}\right),\left(\mathrm{a}_{4}\right),\left(\mathrm{a}_{5}\right)$ and $\left(\mathrm{a}_{6}\right)$ are type B. There are still four diagrams of type B missing: the first is obtained when unwrapping the full four-particle vertex of (b) by substituting its SD series, retaining only the term where the corresponding diagram (i) of Fig 5 appears; the second diagram emerges when unwrapping the full four particle vertex of (v), keeping the term in which diagram (n) of Fig 5 appears; finally, the remaining two diagrams of type B come from diagram (c), after 


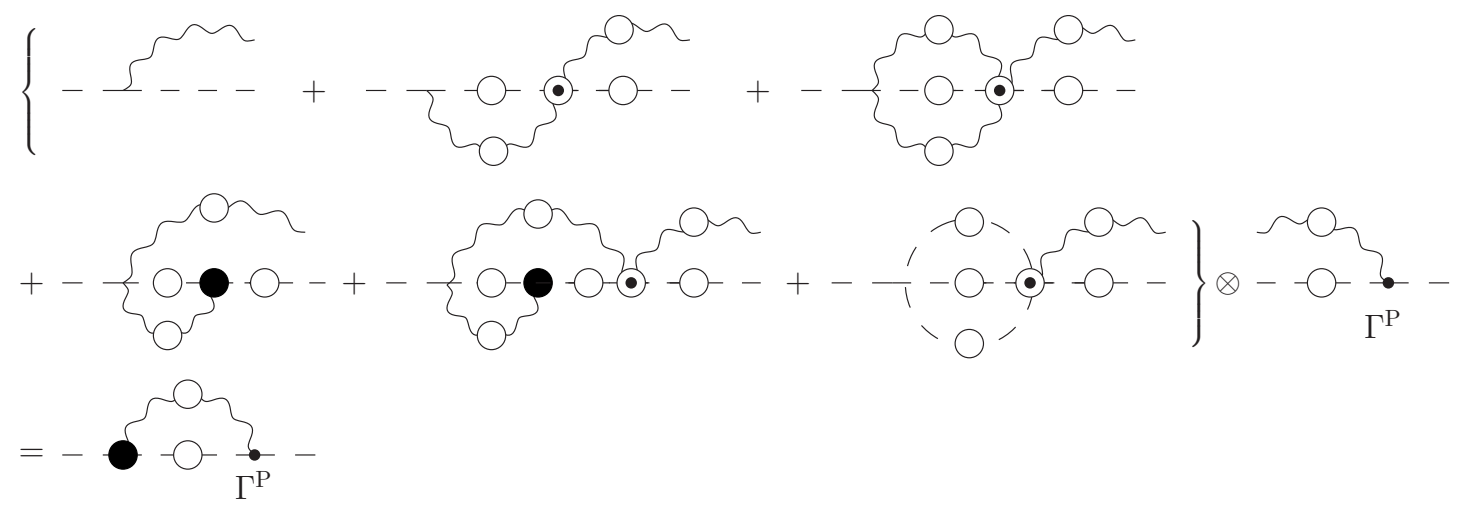

FIG. 8: The rearrangement of $\Gamma^{\mathrm{P}} \Gamma$ terms leading to a $\Gamma^{\mathrm{P}}$ acting on a full $\Gamma$ vertex.

unwrapping the full trilinear vertex on the right, retaining from the corresponding SDE the tree-level vertex and the diagram denoted by (d). All other diagrams contributing to the scalar propagator are of type C, i.e., inert as far as the PT construction is concerned, and will be left untouched. We emphasize that the above separation of diagrams in types $\mathrm{A}, \mathrm{B}$, and $\mathrm{C}$ is unique and unambiguous, regardless of the possibility that one has to further unwrap some of the full internal vertices, using their corresponding SDE. For example, the full internal vertex $\Gamma_{\mu}$ appearing inside diagram $\left(a_{4}\right)$ may be replaced by the r.h.s. of the SDE in Fig.5, forcing the appearance of a bare (tree-level) scalar-scalar-photon vertex. This latter vertex is, however, internal, i.e. all its legs are irrigated by virtual momenta; therefore, it is not supposed to undergo the PT decomposition, and must remain as it is. (For the same reason, in the two-loop construction of section III, the internal vertices in graphs (3a),(3c), and (3d) did not furnish any pinching momenta).

At this point, one carries out on the above type A and B diagrams the usual PT decomposition given in Eqs.(3.2) and (3.4). This will generate the following terms [we use hereafter the notation introduced previously in Eq.(3.7)]

$$
\begin{aligned}
& (x)=(x)^{\mathrm{FF}}+(\mathrm{x})^{\mathrm{P} 0}+(\mathrm{x})^{0 \mathrm{P}}-(\mathrm{x})^{\mathrm{PP}} \quad \mathrm{x}=\mathrm{a}_{1}, \mathrm{a}_{2} \\
& (\mathrm{y})=(\mathrm{y})^{\mathrm{F}}+(\mathrm{y})^{\mathrm{P}} \quad \mathrm{y}=\mathrm{a}_{3}, \mathrm{a}_{4}, \mathrm{a}_{5}, \mathrm{a}_{6}, \mathrm{~b}_{1}, \mathrm{c}_{1}, \mathrm{c}_{2}, \mathrm{v}_{1} .
\end{aligned}
$$

We will now analyze separately the terms appearing in the above equations. 


\section{1. $\Gamma^{\mathrm{P}} \Gamma^{(0)}$ and $\Gamma^{(0)} \Gamma^{\mathrm{P}}$ terms}

The first diagrams we will consider are those of type B. As already discussed, the strategy for treating these diagrams consists in factoring out the $\Gamma^{\mathrm{P}}$ vertex, and choosing the appropriate combination of graphs, in order to force the appearance of a full vertex $\Gamma$ on the opposite side of the diagram. The longitudinal momentum of the pinching vertex will act on this latter full vertex, thus triggering the corresponding WI.

In the case of the $\Gamma^{\mathrm{P}} \Gamma$ the combination one needs to consider is promptly found to be $\left(\mathrm{a}_{1}\right)^{\mathrm{P} 0}+\left(\mathrm{a}_{2}\right)^{\mathrm{P} 0}+\left(\mathrm{a}_{3}\right)^{\mathrm{P}}+\left(\mathrm{a}_{4}\right)^{\mathrm{P}}+\left(\mathrm{a}_{5}\right)^{\mathrm{P}}+\left(\mathrm{a}_{6}\right)^{\mathrm{P}}$; it gives rise to the desired full vertex $\Gamma$, on which the $\Gamma^{\mathrm{P}}$ will act. Specifically,

$$
\begin{aligned}
& \left(\mathrm{a}_{1}\right)^{\mathrm{P} 0}+\left(\mathrm{a}_{2}\right)^{\mathrm{P} 0}+\left(\mathrm{a}_{3}\right)^{\mathrm{P}}+\left(\mathrm{a}_{4}\right)^{\mathrm{P}}+\left(\mathrm{a}_{5}\right)^{\mathrm{P}}+\left(\mathrm{a}_{6}\right)^{\mathrm{P}} \\
= & \int[d k] i \Gamma_{\mu}^{\mathrm{P}}(-k-q, q) i \Delta^{\mu \nu}(k) i S(k+q) i \Gamma_{\nu}(-q, k+q) \\
= & -g \int[d k] \frac{1}{k^{2}} S(k+q) k^{\nu} \Gamma_{\nu}(-k,-q) \\
= & -g^{2} \int[d k] \frac{1}{k^{2}} S(k+q)\left[S^{-1}(k+q)-S^{-1}(q)\right] \\
= & -i G(q) S^{-1}(q) .
\end{aligned}
$$

In obtaining the above expression we have used the WI of Eq.(2.27), the SDE for the auxiliary function $G$ of Eq.(2.40), together with the dimensional regularization result of Eq.(3.8).

For the symmetric term $\Gamma \Gamma^{\mathrm{P}}$ the combination one needs to consider is different, and reads $\left(\mathrm{a}_{1}\right)^{0 \mathrm{P}}+\left(\mathrm{a}_{2}\right)^{0 \mathrm{P}}+\left(\mathrm{b}_{1}\right)^{\mathrm{P}}+\left(\mathrm{c}_{1}\right)^{\mathrm{P}}+\left(\mathrm{c}_{2}\right)^{\mathrm{P}}+\left(\mathrm{v}_{1}\right)^{\mathrm{P}}$, as shown in Fig 8 ; however, the result is the same, and the latter combination will give rise to the mirror contribution of the one just calculated. Therefore one has the result

$$
\begin{aligned}
& \left(\mathrm{a}_{1}\right)^{\mathrm{P} 0}+\left(\mathrm{a}_{2}\right)^{\mathrm{P} 0}+\left(\mathrm{a}_{3}\right)^{\mathrm{P}}+\left(\mathrm{a}_{4}\right)^{\mathrm{P}}+\left(\mathrm{a}_{5}\right)^{\mathrm{P}}+\left(\mathrm{a}_{6}\right)^{\mathrm{P}} \\
+ & \left(\mathrm{a}_{1}\right)^{0 \mathrm{P}}+\left(\mathrm{a}_{2}\right)^{0 \mathrm{P}}+\left(\mathrm{b}_{1}\right)^{\mathrm{P}}+\left(\mathrm{c}_{1}\right)^{\mathrm{P}}+\left(\mathrm{c}_{2}\right)^{\mathrm{P}}+\left(\mathrm{v}_{1}\right)^{\mathrm{P}}=-2 i G(q) S^{-1}(q) .
\end{aligned}
$$

\section{2. $\Gamma^{\mathrm{P}} \Gamma^{\mathrm{P}}$ terms}

Type A diagrams $\left(\mathrm{a}_{1}\right)^{\mathrm{PP}}$ and $\left(\mathrm{a}_{2}\right)^{\mathrm{PP}}$ are of central importance in our construction, since, among other things, they must generate dynamically the BFM ghost sector, exactly as happened in the two-loop example of section III. 


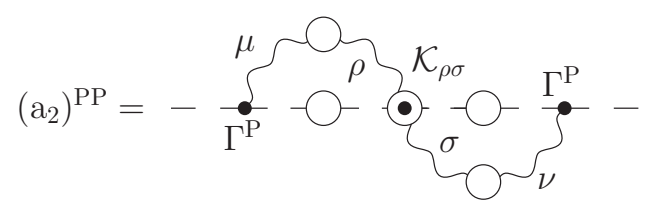

FIG. 9: Feynman diagram corresponding to the term $\left(\mathrm{a}_{2}\right)^{\mathrm{PP}}$.

Of these two diagrams, $\left(a_{1}\right)^{\mathrm{PP}}$ gives simply

$$
\begin{aligned}
\left(\mathrm{a}_{1}\right)^{\mathrm{PP}} & =\int[d k] i \Gamma_{\mu}^{\mathrm{P}}(-k-q, q) i \Delta^{\mu \nu}(k) i S(k+q) i \Gamma_{\nu}^{\mathrm{P}}(-q, k+q) \\
& =-g^{2} \int[d k] S(k)=\bigcup_{\gamma}, \mathrm{O}_{-}
\end{aligned}
$$

and thus generates an effective seagull-like contribution; it will be combined later with

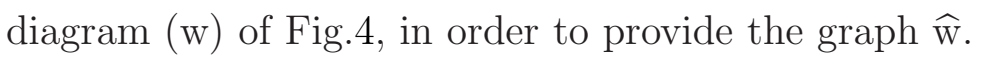

The second term, $\left(\mathrm{a}_{2}\right)^{\mathrm{PP}}$, is shown in Fig 9, and reads

$$
\begin{aligned}
\left(\mathrm{a}_{2}\right)^{\mathrm{PP}} & =\int[d \ell] \int[d k] i \Gamma_{\mu}^{\mathrm{P}}(-\ell-q, q) i \Delta^{\mu \rho}(\ell) i S(\ell+q) i \mathcal{K}_{\rho \sigma}(k,-k-q, \ell+q) \\
& \times i S(k+q) i \Delta^{\sigma \nu}(k) i \Gamma_{\nu}^{\mathrm{P}}(-q, k+q) \\
& =-i g^{2} \int[d \ell] \int[d k] \frac{1}{\ell^{2}} \frac{1}{k^{2}} S(\ell+q) S(k+q) \ell^{\rho} k^{\sigma} \mathcal{K}_{\rho \sigma}(k,-k-q, \ell+q) .
\end{aligned}
$$

We thus see how the SD kernel $\mathcal{K}_{\rho \sigma}$, studied in detail in Section $\nabla$, makes its appearance. Using the WI of Eq.(5.16), and the SDE for the auxiliary function $G$ of Eq.(2.40), we find

$$
\begin{aligned}
\left(\mathrm{a}_{2}\right)^{\mathrm{PP}} & =i g^{4} \int[d \ell] \int[d k] \frac{1}{\ell^{2}} \frac{1}{k^{2}} S(\ell+k+q)-i g^{4} \int[d \ell] \int[d k] \frac{1}{\ell^{2}} \frac{1}{k^{2}} S(\ell+q) S(k+q) S^{-1}(q) \\
& =-\begin{array}{c}
c \\
\vdots
\end{array}-\bigcirc-\cdots
\end{aligned}
$$

where we have recognized that the first term on the RHS of (6.6) is exactly the one needed to generate the BFM ghost sector, whereas the second contributes to the non-perturbative BQI of (2.39). Actually, it is instructive to recognize that this latter term is intimately connected with the perturbative rearrangement of the 1PR diagrams, referred to as "strings", i.e. all possible products of lower order self-energies, appearing when expanding perturbatively $\Delta_{\mu \nu}$ to a given order. In [28, 44] it has been shown that in QCD, the terms one needs to add to convert a string of order $n$ (in $g^{2}$ ) containing more than three self-energy insertions, into a PT string, will be canceled by other strings of the same order, but containing a different 
number of insertions; the only case where this cancellation will not take place is when the string has exactly two $\left(\mathbb{S}_{2}^{(n)}\right)$ or three $\left(\mathbb{S}_{3}^{(n)}\right)$ self-energy insertions. Specializing these results to the case at hand, one has

$$
\begin{aligned}
& \mathbb{S}_{2}^{(n)} \rightarrow \hat{\mathbb{S}}_{2}^{(n)}+2 \sum_{i=1}^{n} G^{(n-i)} S^{-1,(i)}+\sum_{i=1}^{n} G^{(n-i)} S^{-1,(0)} G^{(i)}+4 \sum_{i=2}^{n-1} \sum_{j=1}^{i-1} G^{(n-i)} S^{-1,(j)} G^{(i-j)} \\
& \mathbb{S}_{3}^{(n)} \rightarrow \hat{\mathbb{S}}_{3}^{(n)}-3 \sum_{i=2}^{n-1} \sum_{j=1}^{i-1} G^{(n-i)} S^{-1,(j)} G^{(i-j)}
\end{aligned}
$$

and therefore the contribution coming from the string conversion that must be added to the PT self-energy will be

$$
\mathbb{S}^{(n)}=2 \sum_{i=1}^{n} G^{(n-i)} S^{-1,(i)}+\sum_{i=1}^{n-1} \sum_{j=0}^{i-1} G^{(n-i)} S^{-1,(j)} G^{(i-j)},
$$

or, to all orders,

$$
\mathbb{S}=2 G S^{-1}+G^{2} S^{-1}
$$

Of these two terms the first will cancel against an equal (but opposite in sign) contribution coming from the usual PT construction carried out on the 1PI diagrams, while the second one - which, up to an immaterial $i$ factor is the second term of Eq.(6.6) - represents the genuine string contribution.

\section{The $\Gamma^{\mathrm{F}} \Gamma^{\mathrm{F}}$ terms and the final rearrangement}

We finally consider the $\Gamma^{\mathrm{F}} \Gamma^{\mathrm{F}}$, i.e., $\left(\mathrm{a}_{1}\right)^{\mathrm{FF}},\left(\mathrm{a}_{2}\right)^{\mathrm{FF}},\left(\mathrm{a}_{3}\right)^{\mathrm{F}},\left(\mathrm{a}_{4}\right)^{\mathrm{F}},\left(\mathrm{a}_{5}\right)^{\mathrm{F}},\left(\mathrm{a}_{6}\right)^{\mathrm{F}},\left(\mathrm{b}_{1}\right)^{\mathrm{F}},\left(\mathrm{c}_{1}\right)^{\mathrm{F}}$, $\left(\mathrm{c}_{2}\right)^{\mathrm{F}}\left(\mathrm{v}_{1}\right)^{\mathrm{F}}$.

First of all, notice that in the BFM the only Feynman rules different from the normal $R_{\xi}$ ones (excluding the ghost sector) are those involving the $\widehat{\phi} A_{\mu} \phi^{\dagger}$ and $\widehat{\phi} \widehat{\phi}^{\dagger} \phi \phi^{\dagger}$ vertices; for them we have (see also the Appendix)

$$
\begin{aligned}
& i \Gamma_{\widehat{\phi} A_{\mu} \phi^{\dagger}}\left(q,-p_{1}\right)=2 i g p_{2 \mu}=i \Gamma^{\mathrm{F}}\left(q,-p_{1}\right), \\
& i \Gamma_{\widehat{\phi} \widehat{\phi}^{\dagger} \phi \phi^{\dagger}}\left(k, p_{1}, p_{2}\right)=i\left(\lambda-g^{2}\right) .
\end{aligned}
$$

Now, one should realize that our procedure has systematically replaced all of the scalarscalar-photon vertices with $\Gamma^{\mathrm{F}}$, effectively converting (as far as the Feynman rules describing their interactions are concerned) the external legs, $\phi$ and $\phi^{\dagger}$, into background ones, $\widehat{\phi}$ and 
$\widehat{\phi}^{\dagger}$. Since for the remaining diagrams the external legs can be converted into background ones for free, we find

$$
\left(\mathrm{a}_{1}\right)^{\mathrm{FF}}+\left(\mathrm{a}_{2}\right)^{\mathrm{FF}}+\left(\mathrm{a}_{3}\right)^{\mathrm{F}}+\left(\mathrm{a}_{4}\right)^{\mathrm{F}}+\left(\mathrm{a}_{5}\right)^{\mathrm{F}}+\left(\mathrm{a}_{6}\right)^{\mathrm{F}}=(\widehat{\mathrm{a}})
$$

and

$$
\begin{aligned}
& \left(\mathrm{b}_{1}\right)^{\mathrm{F}}+\ldots=(\widehat{\mathrm{b}}), \\
& \left(\mathrm{c}_{1}\right)^{\mathrm{F}}+\left(\mathrm{c}_{2}\right)^{\mathrm{F}}+\ldots=(\widehat{\mathrm{c}}), \\
& \left(\mathrm{v}_{1}\right)^{\mathrm{F}}+\ldots=(\widehat{\mathrm{v}})
\end{aligned}
$$

where the ellipses denote all other terms appearing in the corresponding SDEs (i.e., the missing terms on the RHS of the second and third line of Fig.7). Summarizing, the PT procedure has enforced the following identity [recall that the $\Gamma^{\mathrm{P}} \Gamma^{\mathrm{P}}$ terms appear with an extra minus sign, see Eq.(3.4)]

$$
\begin{aligned}
i S^{-1}(q) & =-2 i G(q) S^{-1}(q)-i G^{2}(q) S^{-1}(q) \\
& +g^{2} \int[d k] S(k)+i g^{4} \int[d \ell] \int[d k] \frac{1}{\ell^{2}} \frac{1}{k^{2}} S(\ell+k+q) \\
& +(\widehat{\mathrm{a}})+(\widehat{\mathrm{b}})+(\widehat{\mathrm{c}})+(\widehat{\mathrm{v}})+(\mathrm{w})+(\mathrm{z}) .
\end{aligned}
$$

The first two terms corresponds to the last two of the BQI of Eq.(2.39). The third term can be added to the diagram $(\mathrm{w})$ to get

$$
(\mathrm{w})+g^{2} \int[d k] S(k)=i\left(\lambda-g^{2}\right) \int[d k] i S(k)=(\widehat{\mathrm{w}}) .
$$

Moreover

while, finally, $(\mathrm{z}) \equiv(\widehat{\mathrm{z}})$.

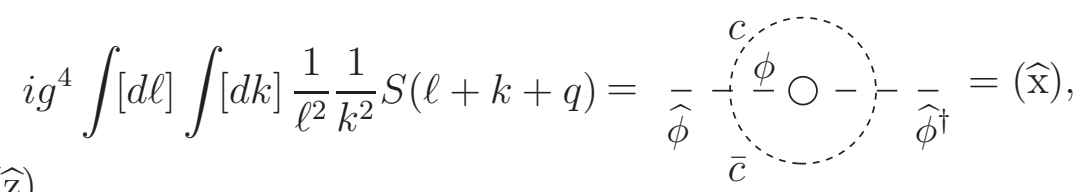

Therefore, we find

$$
\begin{aligned}
i S^{-1}(q) & =-2 i G(q) S^{-1}(q)-i G^{2}(q) S^{-1}(q)+\{(\widehat{\mathrm{a}})+(\widehat{\mathrm{b}})+(\widehat{\mathrm{c}})+(\widehat{\mathrm{v}})+(\widehat{\mathrm{w}})+(\widehat{\mathrm{x}})+(\widehat{\mathrm{z}})\} \\
& =-2 i G(q) S^{-1}(q)-i G^{2}(q) S^{-1}(q)+i \widehat{S}^{-1}(q)
\end{aligned}
$$

which is exactly the BQI for the scalar self-energy. 


\section{B. The trilinear vertex}

The construction of the PT vertices is in general significantly easier than the one carried out for the scalar propagator, due to the fact that there are no type A diagrams. In particular, for the case of the trilinear vertex $\Gamma_{\mu}$ only one type $\mathrm{B}$ diagram needs to be taken into account, namely diagram (d) of Fig.5. One has then

$$
\begin{aligned}
(\mathrm{d})^{\mathrm{P}} & =\int[d \ell] i \Gamma_{\nu}^{\mathrm{P}}(-q-\ell, q) i \Delta_{\nu \rho}(\ell) i S(q+\ell) i \mathcal{K}_{\rho \mu}\left(k, p_{1}, \ell+q\right) \\
& =-g \int[d \ell] \frac{1}{\ell^{2}} S(q+\ell) \ell^{\rho} \mathcal{K}_{\rho \mu}\left(k, p_{1}, \ell+q\right) \\
& =g^{2} \int[d \ell] \frac{1}{\ell^{2}} S(q+\ell) \Gamma_{\mu}\left(p_{1},-k-p_{1}\right) \\
& -g^{2} \int[d \ell] \frac{1}{\ell^{2}} S(\ell+q) S(k+\ell+q) \Gamma_{\mu}(-k-\ell-q, \ell+q) S^{-1}\left(p_{1}\right) \\
& =-i G(q) \Gamma_{\mu}\left(p_{1}, q\right)-i G_{\mu}\left(q, p_{1}\right) S^{-1}\left(p_{1}\right),
\end{aligned}
$$

where we have used the WI of Eq.(5.15). All other diagrams appearing in the SDE for the trilinear vertex are of type $\mathrm{C}$, and remain unchanged; in that sense one can freely replace the external scalar leg with a background one, to get

$$
\begin{aligned}
i \Gamma_{\mu}\left(p_{1}, q\right) & =-i G(q) \Gamma_{\mu}\left(p_{1}, q\right)-i G_{\mu}\left(q, p_{1}\right) S^{-1}\left(p_{1}\right)+\left\{(\mathrm{d})^{\mathrm{F}}+(\widehat{\mathrm{e}})+(\widehat{\mathrm{f}})+(\widehat{\mathrm{g}})+(\widehat{\mathrm{h}})\right\} \\
& =-i G(q) \Gamma_{\mu}\left(p_{1}, q\right)-i G_{\mu}\left(q, p_{1}\right) S^{-1}\left(p_{1}\right)+i \widehat{\Gamma}_{\mu}\left(p_{1}, q\right)
\end{aligned}
$$

which is exactly the BQI of Eq.(2.43).

\section{The quadrilinear vertex}

As the last step we will construct the PT quadrilinear vertex. Again, we have only one type $\mathrm{B}$ diagram that can give pinching contributions, namely diagram (i); its longitudinal momentum acting on the five particle kernel $\mathcal{K}_{\rho \mu \nu}$ will trigger the corresponding WI, Eq.(5.21). One has

$$
\begin{aligned}
(\mathrm{i})^{\mathrm{P}} & =\int[d \ell] i \Gamma_{\nu}^{\mathrm{P}}(-q-\ell, q) i \Delta_{\nu \rho}(\ell) i S(q+\ell) i \mathcal{K}_{\rho \mu \nu}\left(k_{1}, k_{2}, p_{1}, \ell+q\right) \\
& =-g \int[d \ell] \frac{1}{\ell^{2}} S(q+\ell) \ell^{\rho} \mathcal{K}_{\rho \mu \nu}\left(k, p_{1}, \ell+q\right)=\sum_{m=1}^{4}(\mathrm{i})_{m}^{\mathrm{P}}
\end{aligned}
$$


Each $(\mathrm{i})_{m}^{\mathrm{P}}$ represents a term in the WI of Eq.(5.21) which reads

$$
\begin{aligned}
(\mathrm{i})_{1}^{\mathrm{P}} & =g^{2} \int[d \ell] \frac{1}{\ell^{2}} S(\ell+q) \Gamma_{\mu \nu}\left(k_{2}, p_{1}, q\right)=-i G(q) \Gamma_{\mu \nu}\left(k_{2}, p_{1}, q\right) \\
(\mathrm{i})_{2}^{\mathrm{P}} & =g^{2} \int[d \ell] \frac{1}{\ell^{2}} S(\ell+q) S\left(p_{1}-\ell\right) \mathcal{C}_{\mu \nu}\left(k_{2}, p_{1}-\ell, \ell+q\right) S^{-1}\left(p_{1}\right) \\
& =-i G_{\mu \nu}\left(k_{2}, \ell+q, p_{1}\right) S^{-1}\left(p_{1}\right) \\
(\mathrm{i})_{3}^{\mathrm{P}} & =-g^{2} \int[d \ell] \frac{1}{\ell^{2}} S(\ell+q) \Gamma_{\mu}\left(-k_{1}-\ell-q, \ell+q\right) S\left(k_{1}+\ell+q\right) \Gamma_{\nu}\left(p_{1},-p_{1}-k_{2}\right) \\
& =-i G_{\mu}\left(q, k_{2}+p_{1}\right) \Gamma_{\nu}\left(p_{1},-p_{1}-k_{2}\right) \\
(\mathrm{i})_{4}^{\mathrm{P}} & =-g^{2} \int[d \ell] \frac{1}{\ell^{2}} S(\ell+q) \Gamma_{\nu}\left(-k_{2}-\ell-q, \ell+q\right) S\left(k_{2}+\ell+q\right) \Gamma_{\mu}\left(p_{1},-p_{1}-k_{1}\right) \\
& =-i G_{\nu}\left(q, k_{1}+p_{1}\right) \Gamma_{\mu}\left(p_{1},-p_{1}-k_{1}\right) .
\end{aligned}
$$

For all the other (type C) diagrams appearing in the SDEs for the quadrilinear vertex, one can replace the external $\phi$ line with the corresponding background one $\widehat{\phi}$ without introducing new terms in the equation. Using the results above, we finally arrive at the following equation

$$
\begin{aligned}
i \Gamma_{\mu \nu}\left(k_{2}, p_{1}, q\right) & =-i G(q) \Gamma_{\mu \nu}\left(k_{2}, p_{1}, q\right)-i G_{\mu \nu}\left(k_{2}, \ell+q, p_{1}\right) S^{-1}\left(p_{1}\right) \\
& -i G_{\mu}\left(q, k_{2}+p_{1}\right) \Gamma_{\nu}\left(p_{1},-p_{1}-k_{2}\right)-i G_{\nu}\left(q, k_{1}+p_{1}\right) \Gamma_{\mu}\left(p_{1},-p_{1}-k_{1}\right) \\
& \left.\left.+(\mathrm{i})^{\mathrm{F}}+\widehat{\mathrm{j}}\right)+(\widehat{\mathrm{k}})+\widehat{\mathrm{l}}\right)+(\widehat{\mathrm{m}}) \\
& =-i G(q) \Gamma_{\mu \nu}\left(k_{2}, p_{1}, q\right)-i G_{\mu \nu}\left(k_{2}, \ell+q, p_{1}\right) S^{-1}\left(p_{1}\right) \\
& -i G_{\mu}\left(q, k_{2}+p_{1}\right) \Gamma_{\nu}\left(p_{1},-p_{1}-k_{2}\right)-i G_{\nu}\left(q, k_{1}+p_{1}\right) \Gamma_{\mu}\left(p_{1},-p_{1}-k_{1}\right) \\
& +i \widehat{\Gamma}_{\mu \nu}\left(k_{2}, p_{1}, q\right),
\end{aligned}
$$

which is exactly the BQI satisfied by the quadrilinear vertex, Eq.(2.47).

\section{Renormalization issues}

So far we have succeeded in converting the original SD series into an equivalent one, where the external fields have been substituted by their background counterparts. The procedure used has been divided in two steps: $(i)$ carry out the PT algorithm on the (bare) SD series and (ii) compare the result with the BQI satisfied by the (bare) Green's function under scrutiny. A question that rises naturally in this context is whether this entire procedure is preserved by renormalization.

To answer this question, one should realize that the BQIs are a direct consequence of the original BRST symmetry of the theory; therefore, within a suitable regularization scheme, 
such as dimensional regularization, they will be preserved by renormalization, for the same reason that the STIs do not get deformed. Notice that this is completely different from the case of the Nielsen identities [41], describing the gauge fixing parameter dependence of the bare Green's functions. In this latter case, one needs to extend the BRST symmetry to include the variation of the gauge fixing parameter. This, in turn, will spoil the original BRST invariance of the theory, implying that the latter identities get deformed by renormalization already at the one-loop level [45].

To study an explicit example on how renormalization works for the BQIs, let us consider the renormalization of the two point function. On the one hand we clearly have

$$
\begin{aligned}
& S_{\mathrm{R}}^{-1}\left(g_{\mathrm{R}}, \lambda_{\mathrm{R}}, m_{\mathrm{R}}^{2} ; \mu\right)=Z_{\phi} S^{-1}\left(g, \lambda, m^{2} ; \mu, \epsilon\right) \\
& \widehat{S}_{\mathrm{R}}^{-1}\left(g_{\mathrm{R}}, \lambda_{\mathrm{R}}, m_{\mathrm{R}}^{2} ; \mu\right)=Z_{\widehat{\phi}} \widehat{S}^{-1}\left(g, \lambda, m^{2} ; \mu, \epsilon\right) .
\end{aligned}
$$

On the other hand, the function $\widetilde{G} \equiv 1+G$ (where the 1 should be considered as its tree-level value, $\widetilde{G}^{(0)} \equiv 1$ ) renormalizes multiplicatively as

$$
\widetilde{G}_{\mathrm{R}}\left(g_{\mathrm{R}}, \lambda_{\mathrm{R}}, m_{\mathrm{R}}^{2} ; \mu\right)=Z_{\widetilde{G}} \widetilde{G}\left(g, \lambda, m^{2} ; \mu, \epsilon\right) .
$$

Notice, however, that $Z_{\widetilde{G}}$ will not be an independent renormalization constant, because, due to the BQI of Eq.(2.39), its value is determined in terms of $Z_{\widehat{\phi}}$ and $Z_{\phi}$; specifically,

$$
Z_{\widetilde{G}}=Z_{\widehat{\phi}}^{\frac{1}{2}} Z_{\phi}^{-\frac{1}{2}}
$$

To check the validity of this result at lowest order, we can carry out explicitly, at one-loop, the renormalization program for the relevant two-point functions.

The one-loop expansion of Eqs. $(6.22)$ and (6.23) reads

$$
\begin{aligned}
& \Sigma_{\mathrm{R}}^{(1)}\left(g_{\mathrm{R}}, \lambda_{\mathrm{R}}, m_{\mathrm{R}}^{2} ; \mu\right)=\Sigma_{\mathrm{R}}^{(1)}\left(g_{\mathrm{R}}, \lambda_{\mathrm{R}}, m_{\mathrm{R}}^{2} ; \mu, \epsilon\right)+Z_{\phi}^{(1)}\left(q^{2}-m_{\mathrm{R}}^{2}\right)-Z_{m^{2}}^{(1)} m_{\mathrm{R}}^{2} \\
& \widehat{\Sigma}_{\mathrm{R}}^{(1)}\left(g_{\mathrm{R}}, \lambda_{\mathrm{R}}, m_{\mathrm{R}}^{2} ; \mu\right)=\widehat{\Sigma}_{\mathrm{R}}^{(1)}\left(g_{\mathrm{R}}, \lambda_{\mathrm{R}}, m_{\mathrm{R}}^{2} ; \mu, \epsilon\right)+Z_{\widehat{\phi}}^{(1)}\left(q^{2}-m_{\mathrm{R}}^{2}\right)-\widehat{Z}_{m^{2}}^{(1)} m_{\mathrm{R}}^{2} \\
& \widetilde{G}_{\mathrm{R}}^{(1)}\left(g_{\mathrm{R}}, \lambda_{\mathrm{R}}, m_{\mathrm{R}}^{2} ; \mu\right)=\widetilde{G}^{(1)}\left(g, \lambda, m^{2} ; \mu, \epsilon\right)+Z_{\widetilde{G}},
\end{aligned}
$$

while the one-loop divergent parts for the quantum and background two point functions, and the auxiliary function $G$, are given by

$$
\begin{aligned}
& \Sigma^{(1)}\left(g, \lambda, m^{2} ; \mu, \epsilon\right)=-\frac{4}{(4 \pi)^{2} \epsilon} g^{2} q^{2}-\frac{2}{(4 \pi)^{2} \epsilon} m^{2}\left(\lambda+g^{2}\right)+\ldots \\
& \widehat{\Sigma}^{(1)}\left(g, \lambda, m^{2} ; \mu, \epsilon\right)=-\frac{8}{(4 \pi)^{2} \epsilon} g^{2} q^{2}-\frac{2}{(4 \pi)^{2} \epsilon} m^{2}\left(\lambda-g^{2}\right)+\ldots \\
& \widetilde{G}^{(1)}\left(g, \lambda, m^{2} ; \mu, \epsilon\right)=-\frac{2}{(4 \pi)^{2} \epsilon} g^{2}+\ldots,
\end{aligned}
$$


where the ellipses denote finite parts. Combining these results we get

$$
\begin{aligned}
& Z_{\phi}^{(1)}=\frac{1}{4 \pi^{2} \epsilon}, \quad Z_{\widehat{\phi}}^{(1)}=\frac{1}{2 \pi^{2} \epsilon} g^{2}, \\
& Z_{m^{2}}^{(1)} \equiv \widehat{Z}_{m^{2}}^{(1)}=-\frac{1}{8 \pi^{2}}\left(\lambda+3 g^{2}\right), \quad Z_{\widetilde{G}}^{(1)}=\frac{1}{8 \pi^{2} \epsilon} g^{2},
\end{aligned}
$$

which shows that

$$
Z_{\widetilde{G}}^{(1)}=\frac{1}{2}\left(Z_{\widehat{\phi}}^{(1)}-Z_{\phi}^{(1)}\right)
$$

as expected. We end by noticing that the equality between the one-loop mass renormalization constants for quantum and background scalar was to be expected, at least due to two reasons: $(i)$ the scalar and the PT (background) two-point functions differ by the pinch contributions, which are all proportional to $\left(q^{2}-m^{2}\right)$; $(i i) G$ has engineering dimension zero, and thus can give rise to only one independent renormalization constant.

\section{DISCUSSION AND CONCLUSIONS}

In this article we have presented for the first time the extension of the PT at the level of the SDE. Specifically, we have carried out explicitly the PT procedure for the SDEs governing the dynamics of the two- and three-point functions in scalar QED. This Abelian theory has non-trivial properties under the pinching action, due to the simple fact that, unlike normal QED, the fundamental interaction vertex between a pair of charged scalars and a photon depends on the momentum of the incoming scalars. This in turn activates the pinching procedure, and gives rise to a set of modified effective Green's functions, which coincide with the BFM Green's function computed in the Feynman gauge, to all orders in perturbation theory. The extension of this procedure beyond fixed-order perturbation theory requires certain operational adjustments, as discussed here in detail, but does not introduce additional assumptions. The main result of this paper is that the application of the PT algorithm on the SDEs for the conventional Green's functions in the usual covariant gauges generates dynamically the SDEs governing the BFM Green's functions. This conversion of one set of SDE to another is highly non-trivial, given that the Feynman rules and the associated ghost sector is very different within these two gauge-fixing schemes.

As has been emphasized in the Introduction, the upshot of the PT approach is to eventually furnish a self-consistent truncation scheme for the SDEs of gauge theories. It is therefore important to briefly comment why the new SDEs obtained through pinching are superior 
to the conventional ones, and how one should proceed to solve them. The construction carried out here essentially makes manifest extensive all-order rearrangements between the various terms in the SDEs, giving rise to radically different structures. The ensuing massive cancellations are responsible for the special properties of the new PT Green's functions; instead, in the conventional SDE expansion the consequences of these rearrangments are obscured, or even distorted, by casual truncations of the series. In fact, the advantages of the new Schwinger-Dyson series can be best exemplified in the case of QCD itself. Specifically, one of the most distinct features of the PT-BFM scheme is the special way in which the transversality of the background gluon self-energy is realized. In particular, the study of the non-perturbative, SD-type of equation obeyed by the latter quantity reveals that, by virtue of the Abelian-like WIs satisfied by the vertices involved, the transversality is preserved without the inclusion of ghosts [35]. Thus, gluonic and ghost contributions are separately transverse. Moreover, transversality is enforced without mixing the orders in the usual "dressed-loop" expansion: the "one-loop-dressed" and "two-loop-dressed" sets of diagrams are independently transverse. This is to be contrasted with what happens in the usual gauge-fixing scheme of the covariant renormalizable gauges, where the inclusion of the ghost is crucial for the transversality of the gluon self-energy already at the level of the one-loop perturbative calculation. The importance of this property in the context of SDE is that it allows for a meaningful first approximation: instead of the system of coupled equations involving gluon and ghost propagators, one may consider only the subset containing gluons, without compromising the crucial property of transversality. Turning to the second question, one may proceed to solve the new SDEs following two, conceptually equivalent but operationally distinct, approaches. For example, in the case of the scalar propagator considered in this article, one may continue treating $S(q)$ as the unknown dynamical variable, solve the new SD equation in terms of $S(q)$, substitute $S(q)$ into (2.40) to obtain $G(q)$, and subsequently use the BQI of (2.39) to construct $\widehat{S}(q)$. Alternatively, one may regard from the beginning $\widehat{S}(q)$ as the new dynamical variable, and use (2.39) to substitute everywhere on the r.h.s. of the corresponding $\operatorname{SDE} S(q)$ in favor of $\widehat{S}(q)$. It remains to be seen which of these two approaches will turn out to be logistically more expeditious.

In our opinion the most relevant conceptual contribution of this article is the identification of the precise procedure that must be followed when pinching SDEs, together with the necessary field-theoretic ingredients that one needs to employ. Despite the fact that we 
have restricted our attention to scalar QED in the unbroken phase, the procedure described should carry over, up to some additional book-keeping complications to the broken phase of the theory, i.e. when the scalar field develops a non-vanishing vacuum expectation value, $v$, endowing the photon with a mass and adding a Higgs scalar into the physical spectrum. In that case, the object of interest for the PT construction is the effective propagator of the Higgs boson, $\widehat{\Delta}_{H}$, and the possibility of constructing the non-perturbative version of the (Abelian) Higgs effective charge $v^{2} \widehat{\Delta}_{H}$, presented in [24]. In addition, and more importantly, the present work sets up the stage for the generalization of the method in a non-Abelian context, and especially in QCD.

Turning to this important issue, we expect that, as far as the general methodology is concerned, the extension of this work to the case QCD should go through with no additional modifications. From the technical point of view, however, one needs to overcome several obstacles. In particular, as has become obvious by the analysis presented here, one needs to use the result of the contraction of the pinching momenta on the 1PI three-point functions and kernels. In the cases considered here, the Abelian nature of the theory gave rise to simple expressions for the WI needed, whose derivation, although laborious at times, proceeded following textbook techniques. In the case of QCD the object of central interest will be the gluon self-energy; the upshot of the PT construction will consist in transforming its SDE into the corresponding SDE for a background gluon. As is known from the perturbative all-order construction of the gluon self-energy, one of the necessary ingredients for accomplishing this is the STI for three-gluon vertex, derived in the classic work by Ball and Chiu [46]; in the language of our Abelian theory this STI would be the direct analogue of Eq.(2.27). In addition, however, one needs the STI satisfied by the QCD analogue of the kernel $\mathcal{C}_{\mu \nu}$, namely the 1PI kernel with four off-shell gluons; to the best of our knowledge this result does not exist in the literature. Whereas a derivation using some of the techniques reviewed here, or those of [47], may furnish the analogue of Eq.(5.12) for the four-gluon kernel, it is not clear whether the result will be expressed in terms of quantities (e.g., auxiliary ghost Green's functions) that could be directly connected to those appearing typically in the PT construction. This difficulty may be further compounded by the fact that in QCD the ghost sector is interacting, and therefore the auxiliary functions appearing in the corresponding BQI have a much more complicated structure than the $G, G_{\mu}$, and $G_{\mu \nu}$, defined in Eqs.(2.40), (2.45), and (2.48), respectively. Thus, in the corresponding equations instead of bare ghost 
propagators and vertices we will have fully-dressed ones. In addition, the simple WI of Eq.(2.49) will be most certainly replaced by more involved expressions. Despite the technical complications mentioned above, we believe that the extension of the present work to QCD lies well within our reach, and hope to be able to present it in the near future.

\section{Acknowledgments}

D.B. gratefully acknowledges useful correspondence with A. Quadri. The research of J.P. was supported by Spanish MEC under the grant FPA 2005-01678.

Feynman diagrams have been drawn using JaxoDraw [48]. 


$$
\begin{aligned}
& \underset{\phi}{-\sim} \sim_{\phi^{\dagger}}^{-}=\frac{i}{k^{2}-m^{2}} \\
& \sim A_{A_{\nu}}=-\frac{i}{k^{2}}\left[g_{\mu \nu}-(1-\xi) \frac{k_{\mu} k_{\nu}}{k^{2}}\right]
\end{aligned}
$$
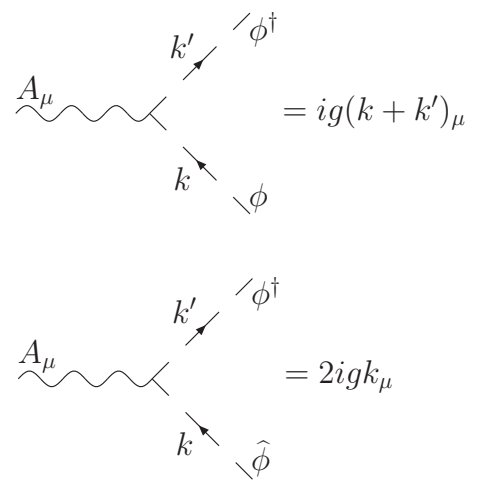
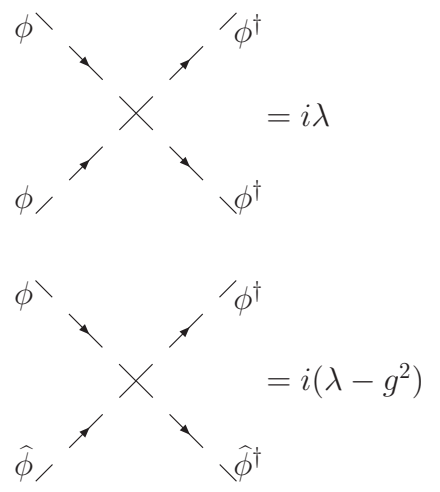
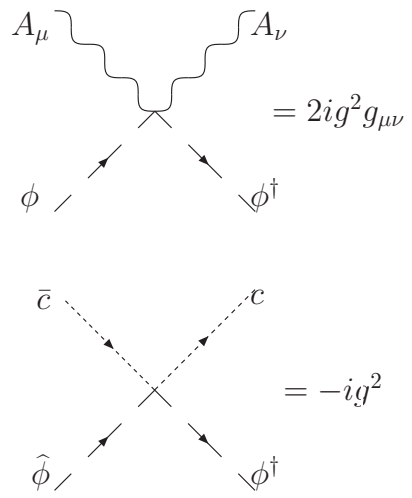

FIG. 10: Feynman rules for scalar QED used in the calculations both in the $R_{\xi}$ as well as the BFM Feynman gauge.

\section{APPENDIX A: FEYNMAN RULES}

The Feynman rules for scalar QED (both in the $R_{\xi}$ and the BFM Feynman gauge) needed for the calculations carried out in the paper are listed in Fig 10. As already put forward in the paper, in order to obtain the full set of Feynman rules in the BFM gauge, one needs not only the gauge-fixing and Faddeev-Popov terms of Eq.(2.14) but also the extra terms coming from the background-quantum splitting $\phi \rightarrow \widehat{\phi}+\phi$ carried out inside the gauge invariant Lagrangian (2.2). The terms in which we are interested reads (in the Feynman gauge)

$$
\widehat{\mathcal{L}} \supset-2 i g A_{\mu}\left(\phi \partial^{\mu} \widehat{\phi}^{\dagger}+\phi^{\dagger} \partial^{\mu} \widehat{\phi}\right)+\left(\lambda-g^{2}\right) \widehat{\phi}^{\dagger} \widehat{\phi} \phi^{\dagger} \phi-g^{2} \bar{c} c\left(\widehat{\phi}^{\dagger} \phi+\widehat{\phi}^{\dagger} \phi\right)
$$

and provides the Feynman rules shown above.

As far as the auxiliary functions are concerned the Feynman rules needed for their calculation can be obtained as follows.

1. For the BRST source terms they can be read directly from the BRST source lagrangian

$$
\mathcal{L}_{\mathrm{BRST}}=\sum_{\Phi} \Phi^{*} s \Phi \supset A_{\mu}^{*} \partial^{\mu} c+i g \phi^{* \dagger} c \phi-i g \phi^{*} c \phi^{\dagger}
$$

Notice that the fact that $A_{\mu}^{*}$ has no interaction other than the one proportional to the derivative of its ghost field shown above, will enforce that the 1PI two point function $\Gamma_{c A_{\mu}^{*}}(q)$ is simply $-q_{\mu}$ to all orders. 

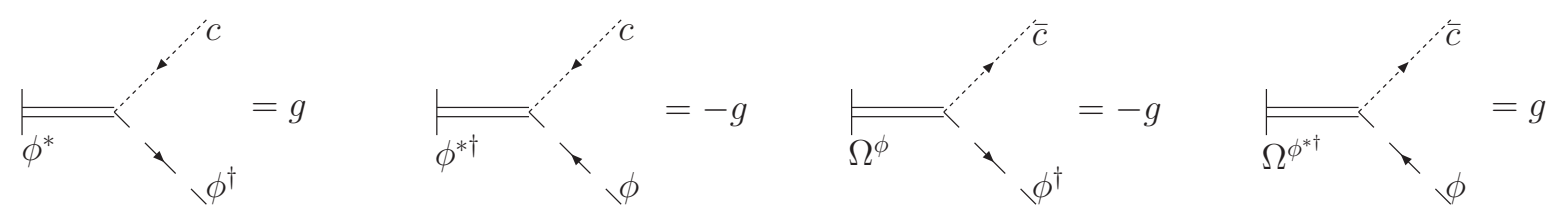

FIG. 11: Feynman rules for the interactions involving BRST and BFM sources.

2. For the background source terms one starts from the general identity

$$
\mathcal{L}_{\mathrm{GF}}+\mathcal{L}_{\mathrm{FPG}}=s \Psi
$$

with $\Psi$ the gauge fixing fermion

$$
\Psi=\bar{c}\left(\frac{\xi}{2} B+\mathcal{F}\right) .
$$

Then if $\mathcal{F}$ is the background gauge fixing function of Eq.(2.12) and we take into account the extended BRST transformations of Eq.(2.32) we get (in the Feynman gauge)

$$
s \Psi \supset i g \bar{c} \Omega^{\phi^{\dagger}} \phi-i g \bar{c} \Omega^{\phi} \phi^{\dagger}
$$

The corresponding Feynman rules for both BRST and background field sources are given in Fig,11.

[1] F. J. Dyson, Phys. Rev. 75, 1736 (1949).

[2] J. S. Schwinger, Proc. Nat. Acad. Sci. 37, 452 (1951); Proc. Nat. Acad. Sci. 37, 455 (1951).

[3] J. M. Cornwall, R. Jackiw and E. Tomboulis, Phys. Rev. D 10, 2428 (1974).

[4] W. J. Marciano and H. Pagels, Phys. Rept. 36, 137 (1978).

[5] C. L. Korpa, J. C. Parikh and P. J. Siemens, Phys. Rev. D 41 (1990) 1276.

[6] V. Sauli and J. Adam, Nucl. Phys. A 689, 467 (2001).

[7] K. i. Kondo, Y. Kikukawa and H. Mino, Phys. Lett. B 220 (1989) 270.

[8] D. C. Curtis and M. R. Pennington, Phys. Rev. D 42, 4165 (1990); Phys. Rev. D 48, 4933 (1993).

[9] D. C. Curtis, M. R. Pennington and D. A. Walsh, Phys. Lett. B 249, 528 (1990).

[10] A. Bashir, A. Kizilersu and M. R. Pennington, Phys. Rev. D 57, 1242 (1998).

[11] V. Sauli, JHEP 0302, 001 (2003). 
[12] S. Mandelstam, Phys. Rev. D 20, 3223 (1979).

[13] A. A. Slavnov, Theor. Math. Phys. 10, 99 (1972) [Teor. Mat. Fiz. 10, 153 (1972)]; J. C. Taylor, Nucl. Phys. B 33, 436 (1971).

[14] A. Salam, Phys. Rev. 130, 1287 (1963); R. Delbourgo and A. Salam, Phys. Rev. 135 (1964) 1398B; R. Delbourgo and P. West, J. Phys. A 10, 1049 (1977); R. Delbourgo, Nuovo Cim. A 49, 484 (1979).

[15] J. M. Cornwall, Phys. Rev. D 26, 1453 (1982).

[16] C. Becchi, A. Rouet and R. Stora, Annals Phys. 98, 287 (1976); I. V. Tyutin, Lebedev Institute Report, 75-39.

[17] J. M. Cornwall and J. Papavassiliou, Phys. Rev. D 40, 3474 (1989).

[18] J. Papavassiliou, Phys. Rev. D 41, 3179 (1990).

[19] D. Binosi and J. Papavassiliou, Phys. Rev. D 65, 085003 (2002).

[20] J. Papavassiliou, Phys. Rev. D 50, 5958 (1994).

[21] J. Papavassiliou, E. de Rafael and N. J. Watson, Nucl. Phys. B 503, 79 (1997).

[22] J. Papavassiliou and A. Pilaftsis, Phys. Rev. Lett. 75, 3060 (1995); Phys. Rev. D 53, 2128 (1996).

[23] J. Papavassiliou and A. Pilaftsis, Phys. Rev. D 54, 5315 (1996).

[24] J. Papavassiliou and A. Pilaftsis, Phys. Rev. Lett. 80, 2785 (1998); Phys. Rev. D 58, 053002 (1998).

[25] M. Binger and S. J. Brodsky, Phys. Rev. D 74, 054016 (2006).

[26] N. Caporaso and S. Pasquetti, JHEP 0604, 016 (2006).

[27] N. Caporaso and S. Pasquetti, arXiv:hep-th/0609168.

[28] D. Binosi and J. Papavassiliou, Phys. Rev. D 66, 111901 (2002); J. Phys. G 30, 203 (2004).

[29] D. Binosi, J. Phys. G 30, 1021 (2004).

[30] B. S. Dewitt, Phys. Rev. 162, 1195 (1967); J. Honerkamp, Nucl. Phys. B 48, 269 (1972); R. E. Kallosh, Nucl. Phys. B 78, 293 (1974); H. Kluberg-Stern and J. B. Zuber, Phys. Rev. D 12, 482 (1975); G. 't Hooft, In *Karpacz 1975, Proceedings, Acta Universitatis Wratislaviensis No.368, Vol.1*, Wroclaw 1976, 345-369; S. Weinberg, Phys. Lett. B 91, 51 (1980); L. F. Abbott, Nucl. Phys. B 185, 189 (1981).

[31] S. Weinberg, The Quantum Theory of Fields (Cambridge University Press, New York, 1996), Vol. II. 
[32] A. Denner, G. Weiglein and S. Dittmaier, Phys. Lett. B 333, 420 (1994); S. Hashimoto, J. Kodaira, Y. Yasui and K. Sasaki, Phys. Rev. D 50, 7066 (1994); J. Papavassiliou, Phys. Rev. D 51, 856 (1995); A. Pilaftsis, Nucl. Phys. B 487, 467 (1997).

[33] J. Papavassiliou and J. M. Cornwall, Phys. Rev. D 44, 1285 (1991).

[34] N. E. Mavromatos and J. Papavassiliou, Phys. Rev. D 60, 125008 (1999).

[35] A. C. Aguilar and J. Papavassiliou, JHEP 0612, 012 (2006)

[36] R. B. Sohn, Nucl. Phys. B 273, 468 (1986); A. Hadicke, JENA-N-88-19.

[37] P. Gambino and P. A. Grassi, Phys. Rev. D 62, 076002 (2000); P. A. Grassi, T. Hurth and M. Steinhauser, Annals Phys. 288, 197 (2001).

[38] D. Binosi and J. Papavassiliou, Phys. Rev. D 66, 025024 (2002).

[39] I. A. Batalin and G. A. Vilkovisky, Phys. Lett. B 69 (1977) 309; Phys. Rev. D 28 (1983) 2567 [Erratum-ibid. D 30 (1984) 508].

[40] J. Papavassiliou, Phys. Rev. Lett. 84, 2782 (2000); Phys. Rev. D 62, 045006 (2000).

[41] N. K. Nielsen, Nucl. Phys. B 101, 173 (1975).

[42] J. D. Bjorken and S. D. Drell, "Relativistic Quantum Field Theory", McGraw-Hill Inc (1965).

[43] C. Itzykson and J. B. Zuber, "Quantum Field Theory", McGraw-Hill Inc (1980).

[44] D. Binosi and J. Papavassiliou, Nucl. Phys. Proc. Suppl. 121, 281 (2003).

[45] For the all-order form of the Nielsen identities after renormalization, see D. Binosi, J. Papavassiliou and A. Pilaftsis, Phys. Rev. D 71, 085007 (2005).

[46] J. S. Ball and T. W. Chiu, Phys. Rev. D 22, 2550 (1980) [Erratum-ibid. D 23, 3085 (1981)].

[47] P. Pascual and R. Tarrach, "QCD: Renormalization For The Practitioner," Lect. Notes Phys. $194(1984) 1$.

[48] D. Binosi and L. Theussl, Comput. Phys. Commun. 161, 76 (2004); (http://jaxodraw.sourceforge.net). 